\title{
Kinesthesia in a Sustained-attention Driving Task
}

\author{
Chun-Hsiang Chuang ${ }^{\mathrm{a}, \mathrm{b}, \mathrm{c}}$, Li-Wei Ko ${ }^{\mathrm{a}, \mathrm{d}}$, Tzyy-Ping Jung ${ }^{\mathrm{c}, \text {, }}$, Chin-Teng Lin ${ }^{\mathrm{a}, \mathrm{b}, \mathrm{e}^{*}}$ \\ ${ }^{a}$ Brain Research Center, National Chiao Tung University, Hsinchu, Taiwan \\ ${ }^{\mathrm{b}}$ Institute of Electrical Control Engineering, Department of Electrical and Computer Engineering, \\ National Chiao Tung University, Hsinchu, Taiwan \\ ${ }^{c}$ Swartz Center for Computational Neuroscience, Institute for Neural Computation, University of \\ California, San Diego, CA, USA \\ ${ }^{\mathrm{d}}$ Department of Biological Science and Technology, National Chiao Tung University, Hsinchu, \\ Taiwan \\ ${ }^{\mathrm{e}}$ Center for Advanced Neurological Engineering, Institute of Engineering in Medicine, University \\ of California, San Diego, CA, USA \\ * Correspondence should be addressed to either of the following: \\ Tzyy-Ping Jung, Institute for Neural Computation, University of California San Diego, 9500 \\ Gilman Dr., La Jolla, CA 92093-0559, USA \\ Phone: +1 (858) 822-7555 \\ E-mail: jung@sccn.ucsd.edu
}

Chin-Teng Lin, Brain Research Center, National Chiao Tung University, No. 1001, University Rd., Hsinchu 30010, Taiwan

Phone: +886 (3) 571-2121 ext 54455

E-mail: ctlin@mail.nctu.edu.tw 


\section{Abstract}

This study investigated the effects of kinesthetic stimuli on brain activities during a sustained-

attention task in an immersive driving simulator. Tonic and phasic brain responses on multiple timescales were analyzed using time-frequency analysis of electroencephalographic (EEG) sources identified by independent component analysis (ICA). Sorting EEG spectra with respect to reaction times (RT) to randomly introduced lane-departure events revealed distinct effects of kinesthetic stimuli on the brain under different performance levels. Experimental results indicated that EEG spectral dynamics highly correlated with performance lapses when driving involved kinesthetic feedback. Furthermore, in the realistic environment involving both visual and kinesthetic feedback, a transitive relationship of power spectra between optimal-, suboptimal-, and poor-performance groups was found predominately across most of the independent components. In contrast to the static environment with visual input only, kinesthetic feedback reduced theta-power augmentation in the central and frontal components when preparing for action and error monitoring, while strengthening alpha suppression in the central component while steering the wheel. In terms of behavior, subjects tended to have a short response time to process unexpected events with the assistance of kinesthesia, yet only when their performance was optimal. Decrease in attentional demand, facilitated by kinesthetic feedback, eventually significantly increased the reaction time in the suboptimal-performance state. Neurophysiological evidence of mutual relationships between behavioral performance and neurocognition in complex task paradigms and experimental environments, presented in this study, might elucidate our understanding of distributed brain 
dynamics, supporting natural human cognition and complex coordinated, multi-joint naturalistic behavior, and lead to improved understanding of brain-behavior relations in operating environments.

Keywords: EEG, kinesthesia, driving, independent component analysis, time-frequency analysis 


\section{Introduction}

Sensory feedback is one of the most important components in sustaining attention (Sarter et al., 2001). With respect to everyday driving, an early report (Gioia and Morphew, 1968) claimed that $90 \%$ of the information that is used by drivers depends on vision, but the accuracy of this estimate was then debated by Sivak (1996). In fact, perception inference is largely automatic (Kasschau, 1985), effectively helping drivers obtain relevant information and enabling them to focus on a given task (Kemeny and Panerai, 2003; Kim et al., 2012). Kinesthesia is an important sensory source. Various degrees of vehicle motion can contribute to the generation of kinesthetic and vestibular sensations that inform drivers about the direction and speed of vehicle motion, its location, and the surrounding environment. Motion cues have been shown to improve driving performance (Kemeny and Panerai, 2003; Wallis et al., 2007). We hypothesize that a kinesthetic input might alter observed brain activity.

Monitoring the neurophysiological activities that are induced by motion in a naturalistic environment using vibration-sensitivity equipment, such as functional magnetic resonance imaging (Friston et al., 1996) or positron emission tomography (Nehmeh and Erdi, 2008), represents a severe measurement challenge. The electroencephalogram (EEG) is currently the clearly preferred device for imaging the brains of humans as they performing tasks that involve natural movements in a real-world environment (Liao et al., 2012).

Recently, many laboratory-based investigations (Banks et al., 2004; Boyle et al., 2008; Campagne et al., 2004; De Rosario et al., 2010; Eoh et al., 2005; Jap et al., 2011; Jap et al., 2009; 
Khushaba et al., 2011; Lal and Craig, 2001; Lal and Craig, 2002; Lal et al., 2003; Lin et al., 2010;

Lin et al., 2006; Lin et al., 2005; Lin et al., 2012b; Otmani et al., 2005) have led to foundational insights into the brain functions that are associated with sustaining attention on the task of safe driving. They demonstrated the feasibility of accurately estimating shifts in a driver's levels of arousal, fatigue, and vigilance, as represented by changes in their task performance, by evaluating EEG changes. Most relevant studies have found an increase in theta power (4-7 Hz)(Campagne et al., 2004; De Rosario et al., 2010; Eoh et al., 2005; Huang et al., 2009; Jap et al., 2011; Jap et al., 2009; Lal and Craig, 2001; Lal and Craig, 2002; Lal et al., 2003; Otmani et al., 2005), the frequency of theta burst (Banks et al., 2004; Eoh et al., 2005) or the duration of episodes of theta activity (Banks et al., 2004; Boyle et al., 2008) as the task performance declined or a progressive deterioration of the driver's attention. Alpha activity $(8-12 \mathrm{~Hz})$ is another useful index of task performance, but it varies among studies. Increases of the alpha power, the density ratio of the sum of theta and alpha to beta, and the density ratio of alpha to beta were observed as the driving error increased (Campagne et al., 2004) or fatigue occurred (Eoh et al., 2005; Jap et al., 2009; Otmani et al., 2005). The aforementioned studies, however, have not quantitatively explored the effects of motion stimuli to human behavior and brain dynamics.

Previous studies showed that the motion and vestibular stimuli elicited an evoked potential over the fronto-central areas such as Fz, Cz, and Pz (Elidan et al., 1991; Loose et al., 1999; Nolan et al., 2012; Probst et al., 1997; Rodionov et al., 1996). The cortical regions involve in the processing of motion/vestibular cues include the posterior parietal cortex, insular, frontal cortex, 
somatosensory cortex, cingulate cortex, and striate/extrastriate cortex (Deutschlander et al., 2002;

Lopez and Blanke, 2011). Furthermore, functional magnetic resonance imaging (fMRI) studies

(Bremmer et al., 2001; Scheef et al., 2009) reveal increased activation at the posterior parietal, premotor cortex and visual cortices when the brain is engaged in processing motion. These brain regions that are observably affected by motion or vestibular stimuli largely overlap with the regions associated with performance lapses. However, the interaction effect of kinesthetic feedback and performance lapse on the brain activity remains unknown.

Therefore, this study thoroughly elucidated how kinesthesia affects brain activity, especially when momentary cognitive lapses were experienced. This study implemented an event-related lanedeparture paradigm (Huang et al., 2009) on a realistic dynamic driving simulator (Lin et al., 2010) to explore detailed EEG dynamics associated with motion cues under different levels of performance. Each subject participated in a simulated driving session without kinesthetic feedback and a separate session with kinesthetic feedback on different days, in which subject' behavior and EEG signals were recorded simultaneously. Owing to volume conduction through the cerebrospinal fluid, skull and scalp, EEG data collected from any point on the scalp may include activity from brain, extra-brain and artifactual sources. This signal-mixing problem reduces the signal-to-noise ratio of desired EEG brain signals, making EEG analysis and interpretation rather challenging tasks (Jung et al., 2001; Makeig et al., 1997). Moreover, as is well known, brain responses to stimulus presentations may vary widely across subjects in both time course and spatial origins. Therefore, equivalent EEG sources across subjects must be identified to assist in an anatomic and functional 
interpretation of the component process. By using independent component analysis (ICA), this study separated the data of each subject into temporally independent components (ICs)(Jung et al., 2001; Makeig et al., 1997). Several studies have demonstrated the efficacy of clustering ICs according to their cortical locations estimated from the scalp topographies (Makeig et al., 2002; Marco-Pallares et al., 2005; Milne et al., 2009; Onton et al., 2005). Thus, based on a source localization/imaging method, this study estimated the cortical locations of individual ICs from their scalp maps by individual columns of the ICA unmixing matrix. ICs obtained from all of the subjects were then grouped into distinct clusters with a high intra-cluster similarity (Delorme and Makeig, 2004) based on commonalities of scalp topographies, equivalent dipole source locations, and timefrequency properties (Delorme and Makeig, 2004). The neural generators of task-relevant oscillatory brain activity were then identified, based on equivalent dipole locations of ICs. Finally, this study compared the EEG spectra of ICs of interest under different experimental conditions (w/ or w/o kinesthetic feedback) at various levels of behavioral performance, as determined by the required time to react to a random lane-departure event. 


\section{Materials and Methods}

\subsection{Experimental environment and paradigm}

This study implemented an event-related lane-departure driving paradigm (Huang et al., 2009) on a dynamic simulator (Lin et al., 2010) to explore detailed EEG dynamics associated with motion cues under different levels of task performance. The immersive virtual reality (VR) scenario (Fig. 1A) simulated nighttime driving at a constant speed of $100 \mathrm{~km} / \mathrm{hr}$ on a four-lane divided highway. The VR program (Fig. 1B) randomly introduced lane-perturbation events to cause the virtual vehicle to drift from the center of the cruising lane, and participants had been instructed to steer the vehicle back to the cruising lane as fast as possible after becoming aware of the deviation. If the subjects did not respond to the lane-perturbation event, falling asleep for example, and then the vehicle could hit the left and right curb of the roadside within $2.5 \mathrm{~s}$ and $1.5 \mathrm{~s}$, respectively. The vehicle would then continue to move along the curb until it returned to the original lane. The intertrial interval was set to 5 10 s. The experiment was begun in the early afternoon (13:00-14:00) after lunch and lasted for about 90min when the circadian rhythm of sleepiness was at its peak (Ferrara and De Gennaro, 2001). Subjects' cognitive states and driving performance were monitored via a surveillance video camera and the vehicle trajectory throughout the experiment.

\subsection{Experimental session with and without a kinesthetic feedback}

To investigate the effect of kinesthesia on brain activity in the sustained-attention driving task, each subject was asked to participate two driving sessions on different days. Each session lasted for 
about 90min. One was the driving session (noted as $\mathrm{K}^{-}$) with a fixed-based simulator but with no kinesthetic feedback, so the subject had to monitor the vehicle deviation visually from the virtual scene. The other driving session (noted as $\mathrm{K}^{+}$) involved a motion-based simulator with a six degreeof-freedom Stewart platform (Fig. 1) to simulate the dynamic response of the vehicle to the deviation event or steering. The visual and kinesthetic inputs together aroused the subject to attend to the deviation event and take action to correct the driving trajectory. The order of the $\mathrm{K}^{-}$session and the $\mathrm{K}^{+}$session was counterbalanced across subjects, i.e., half of the participants performed the $\mathrm{K}^{-}$session first followed by the $\mathrm{K}^{+}$session and the other half of participants performed the $\mathrm{K}^{+}$ session first followed by the $\mathrm{K}^{-}$session. The second session was performed after an average delay of a week.

\subsection{Participants}

Sixteen voluntary participants of age $24.2 \pm 3.7$ (ten males and six females) with normal or corrected-to-normal vision were recruited in this study. None of subjects reported sleep deprivation in the preceding weeks and none had a history of drug abuse according to self-report. Before each experiment, the subjects needed to answer a questionnaire regarding their sleep patterns to make sure they were not sleep deprived or took any medication that might influence their cognitive states.

Every subject was also required to have a normal work and rest cycle, enough sleep (around 8hrs of sleep each night), and not stay up late (no latter than 11:00 pm) a week before the experiment. Additionally, the subjects did not have imbibed alcohol and caffeinated drinks, or participated in 
strenuous exercise a day before the experiments. To evaluate accurately their driving performance, the participants attended a pre-test session to determine that none was afflicted with simulator sickness. The Institutional Review Board of the Veterans General Hospital, Taipei, Taiwan, approved the study. All participants were asked to read and sign an informed consent form before participating in the EEG experiments.

\subsection{Data recording and preprocessing}

The EEG signals were recorded using $\mathrm{Ag} / \mathrm{AgCl}$ electrodes that were attached to a 32-channel Quik-Cap (Compumedical NeuroScan). Thirty electrodes were arranged according to a modified international 10-20 system, and two reference electrodes were placed on both mastoid bones. The skins under the reference electrodes were abraded using Nuprep (Weaver and Co., USA) and disinfected with a $70 \%$ isopropyl alcohol swab before calibration. The impedance of the electrodes was calibrated under $5 \mathrm{k} \Omega$ using NaCl-based conductive gel (Quik-Gel, Neuromedical Supplies®). The EEG signals from the electro-cap were amplified using the Scan NuAmps Express system (Compumedics Ltd., VIC, Australia) and recorded at a sampling rate of $500 \mathrm{~Hz}$ with 16bit quantization. Before the data were analyzed, the raw EEG recordings were preprocessed using a digital band-pass filter $(1-50 \mathrm{~Hz})$ to remove line noise and artifacts and then down-sampled to 250 $\mathrm{Hz}$ to reduce the number of data. Severe contamination of the EEG signal by eye movement, blinking, muscle activity, and environmental noise was manually removed to minimize their effect on the subsequent analysis. Table 1 shows the number of trials collected in two sessions. 


\section{Data Analysis}

\subsection{Independent EEG processes and category}

Independent component analysis (ICA) was applied to EEG signals using the EEGLAB routine (Delorme and Makeig, 2004) to separate out temporally independent time courses of the activation. For each recorded datum, a maximum of 30 independent components (ICs) and their corresponding mixing matrix were decomposed. The ICs, obtained from 16 subjects, were grouped into distinct clusters with high intra-cluster similarity (Delorme and Makeig, 2004) based on commonalities of scalp topographies (2-D visualization maps of the columns of the mixing matrix), equivalent dipole source locations (Oostenveld and Oostendorp, 2002), and event-related spectral perturbations (Delorme and Makeig, 2004). Based on suggestions in the literature (Huang et al., 2008; Huang et al., 2009; Lin et al., 2010), five IC clusters - frontal, central, motor, parietal, and occipital (as displayed in Fig. 2) - were selected as the ICs of interest for further analysis.

The continuous IC time series was then segmented into a set of epochs of varying lengths from $2 \mathrm{~s}$ before each deviation event to the occurrence of the following deviation event. Each extracted epoch comprises four critical periods, which are (1) the baseline period (the period before the onset of the deviation), (2) the lane-departure period (between the onset of the deviation and the onset of the response), (3) the steering period (between the onset and the end of the response), and (4) the post-movement period (between the end of the response and the next deviation onset). 
The response time (RT) to each lane-departure event (i.e., the time between the onset of the deviation and the onset of the response) was used as an objective behavioral measurement to characterize all EEG epochs. Three groups of epochs were defined: the optimal-performance, the suboptimal-performance, and the poor-performance groups. For each subject, the RTs collected from the first 10 minutes of the experiment were used to construct a null distribution of the optimal RTs. To make sure the subjects had optimal performance during the first $10 \mathrm{~min}$ baseline period, both their facial videos and responses to the lane departure events were closely monitored. The experimenters visually observe subjects' facial features such as eye movements (blink rate, blink duration, long closure rate etc.), head pose and gaze direction via the surveillance video to determine whether subjects take eyes off the road. Most importantly, the behavioral data (vehicle trajectory) objectively confirms whether or not the estimated RTs during the first 10 min were short ( $700 \mathrm{~ms})$. Then, for the rest of the experiment, any epoch with RT no greater than $95 \%$ of the values in the null distribution (i.e., it was not significantly different from the optimal RT, p<0.05) was considered to be an epoch of optimal-performance group. The poor-performance epochs were those with RTs exceeding twice of the threshold of the optimal-performance epochs. Finally, the epochs with RTs in between were considered as suboptimal.

\subsection{Event-related time-frequency estimation}

To investigate brain dynamics following the lane-departure events and the subsequent motor responses, each epoch was separately transformed into the time-frequency representation using the 
event-related spectral perturbation routine (Delorme and Makeig, 2004). The mean baseline log power spectrum (in $\mathrm{dB}$ ) of the optimal epochs, used as the reference value, was subtracted from each estimated spectrum. All estimated spectra were then linearly warped to a fixed temporal length. The new latencies of the events, obtained by estimating the medians of the original latencies of the events, were 0s, 0.960s, 3.160s, and 9.407s for the onset of the deviation, the onset of the response, the end of the response, and the onset of the next deviation (end of the epoch), respectively. The total number of time points (flames) that were used to determine the new time-frequency estimate was 1087 , of which the baseline period, the lane-departure period, the steering period, and the postmovement period were associated with 192 pts, 97 pts, 212 pts, and 586 pts, respectively. For each component cluster and each driving session, as presented in Figs. 4A-E, the mean delta- $(\delta: 1-3 \mathrm{~Hz})$, theta- $(\theta: 4-7 \mathrm{~Hz})$, alpha- $(\alpha: 8-12 \mathrm{~Hz})$, and beta- $(\beta: 13-20 \mathrm{~Hz})$ band powers were vertically stacked according to the RT of the epoch, yielding four 2D images of event-related band power dynamics that accompanied the transition of behavioral changes from optimal, suboptimal to poor performance (x-axis: time and y-axis: RT).

\subsection{Statistical analysis}

Linear dependence between the band power and the RT for each component cluster was measured by calculating the Pearson's correlation coefficient $(r)$, as shown in Fig. 4, and correcting for multiple comparisons by using the false discovery rate (FDR)-adjusted $p$-value $<0.001$. The Kolmogorov-Smirnov and Wilcoxon signed-rank tests were also performed on the RTs to determine 
the behavioral differences between $\mathrm{K}^{-}$and $\mathrm{K}^{+}$sessions. Additionally, the effect size was assessed by using the formula: $r=z / \sqrt{ }(N)($ Fritz et al., 2012), where $z$ denotes the Wilcoxon z-statistic and $N$ represents the total number of observations. This study also investigated how event types (baseline, lane-departure, steering, and post-movement), performance quality (optimal, suboptimal, and poor), kinesthetic feedback $\left(\mathrm{K}^{-}\right.$and $\left.\mathrm{K}^{+}\right)$, and their interactions affect power spectra by performing a three-way repeated-measure ANOVA with a $4 \times 3 \times 2$ design for four bands $(\delta, \theta, \alpha$, and $\beta$ ) and five independent clusters. Since 20 ANOVAs were performed independently, multiple comparison corrections were made using the FDR theory. Notably, the band powers across different RTs were averaged into three bins (representing 3 levels of performance quality) for each of the 4 event types and each of the 2 kinesthetic feedback types before performing each ANOVA test. Table 2 lists the ANOVA statistics including the FDR-adjusted $p$-value and the effect size (as measured by partial eta squared, $\eta_{p}^{2}$ ) corresponding to the main effect and the interaction effect. Moreover, the significant interaction effects inferred from Table 2 were further visualized by interaction plots (Figs. 5-7), in which the multiple comparison results were provided as well. Furthermore, post-hoc t-tests were conducted for follow-up analysis in repeated measure ANOVA. The effect size was measured the absolute value of Cohen' $d$. 


\section{Results}

\subsection{Task performance}

Figs. 3A and 3B plot the histograms of the RTs (reddish distribution), the end of the response (greenish distribution), and the onset of the next deviation (bluish distribution) for the $\mathrm{K}^{-}$session and the $\mathrm{K}^{+}$session, respectively. All of the distributions were positively skewed. The sample skewnesses of the onset of the deviation, the onset of the response, and the end of the response were 3.179 (3.662), 2.564 (2.288), and 2.276 (4.942), respectively, for the $\mathrm{K}^{-}\left(\mathrm{K}^{+}\right.$) session. A two-sided two-sample Kolmogorov-Smirnov test was performed to demonstrate how kinesthetic feedback affects RTs. According to those results, the $\mathrm{K}^{-}$and $\mathrm{K}^{+}$sessions $(p<0.001)$ at the onset and end of the response significantly differed in the distributions of event latencies. The maximum difference between the onsets of response in two driving sessions was $115 \mathrm{~ms}$, and that between the ends of the response was $152 \mathrm{~ms}$. Comparing the $\mathrm{RTs}$ in the $\mathrm{K}^{-}$session with those in the $\mathrm{K}^{+}$session showed that subjects performed better in the driving task when they had kinesthetic feedback to integrate with incoming visual information.

In Fig. 3C, all of the epochs are time-locked with the onset of the deviation (leftmost solid line at time $=0$ ), and the times of occurrence of the events were stacked vertically according to the RTs of the epochs in which they occurred. The traces in Fig. 3C represent, from left to right, the onset of the response (dashed trace), the end of the response (dotted-dashed trace), and the onset of the next deviation (solid trace), respectively. The upper and lower bounds of RTs on the optimalperformance and poor-performance groups were $0.756 \mathrm{~s}$ and $1.512 \mathrm{~s}$, respectively. In the optimal- 
performance group, median $\mathrm{RT}$ of the $\mathrm{K}^{+}$sessions $(0.665 \mathrm{~s})$ was statistically significantly shorter than that of the $\mathrm{K}^{-}$sessions $(0.682 \mathrm{~s})$ (Wilcoxon signed-rank test: $\left.p<0.001, r=-0.35\right)$. In the suboptimal-performance group, median $\mathrm{RT}$ of the $\mathrm{K}^{+}$sessions $(1.034 \mathrm{~s})$ was significantly larger than that of the $\mathrm{K}^{-}$sessions $(0.951 \mathrm{~s})(p<0.0009, r=-0.36)$. In the poor-performance group, the difference of median $\mathrm{RT}$ between $\mathrm{K}^{-}(5.743 \mathrm{~s})$ and $\mathrm{K}^{+}(5.022 \mathrm{~s})$ sessions was statistically insignificant $(p=1, r=-0.11)$.

\subsection{Co-variance of event-related band powers with increase of $R T$}

This study also attempted to justify the functional role of event-related spectral dynamics of independent components by using Pearson's correlation to evaluate the dependence between neural dynamics and behavior. Pearson's correlation coefficients $(r)$ shown in Figs. 4 specified the strength of the linear dependence between the band powers and the fluctuations of RTs across time and events (FDR-adjusted $p$-value $<0.001$ ). For the EEG activity during the baseline period, all delta and theta powers exhibited a strongly positive correlation (Pearson's $r>0.5$ ) with changes of the RTs in all clusters under both conditions. The strongly positive correlations between the alpha powers and the RTs were found in the frontal cluster (both $\mathrm{K}^{-}$and $\mathrm{K}^{+}$conditions), central cluster ( $\mathrm{K}^{-}$and $\mathrm{K}^{+}$conditions), parietal cluster $\left(\mathrm{K}^{-}\right.$condition), and occipital cluster ( $\mathrm{K}^{-}$condition). Additionally, a strong positive correlation between the beta powers and the RTs was found in the frontal cluster $\left(\mathrm{K}^{-}\right.$condition) and the central cluster $\left(\mathrm{K}^{-}\right.$and $\mathrm{K}^{+}$conditions $)$. During the lanedeparture period, delta power ( $\mathrm{K}^{-}$condition) of the frontal cluster (Fig. $\left.4 \mathrm{~A}\right)$, delta ( $\mathrm{K}^{-}$condition), 
theta $\left(\mathrm{K}^{-}\right.$condition), and alpha power $\left(\mathrm{K}^{+}\right.$condition) of the central cluster (Fig. 4B), theta power ( $\mathrm{K}^{-}$condition) of the motor cluster (Fig. $\left.4 \mathrm{C}\right)$, delta ( $\mathrm{K}^{-}$condition), theta ( $\mathrm{K}^{+}$condition), and alpha powers (both $\mathrm{K}^{-}$and $\mathrm{K}^{+}$conditions) of the parietal (Fig. 4D) cluster were nearly independent of the RTs. Additionally, the correlation coefficient decreased markedly to Pearson's $r=0$. Except for the delta power of the central cluster, all of the band powers were strongly positively correlated with RTs throughout the lane-departure and steering periods. Following the offset of a response, most of the band powers were highly positively correlated with RTs. However, for alpha powers of the central $\left(\mathrm{K}^{+}\right.$condition only), motor, parietal, and occipital clusters and the beta powers of the motor, parietal, and occipital clusters, the correlation coefficients decreased to zero.

\subsection{Main Effects}

Main effect of event type

Two-dimensional images in Fig. 4 show the RT-sorted event-related spectral perturbation in the frontal, central, motor, parietal, and occipital IC clusters. The main effect of the event type (three-way repeated measures ANOVA, FDR-adjusted $p$-value<0.001)(Table 2) was observed in all EEG spectral bands of the frontal $\left(\delta: F(3,45)=121.17, \eta_{p}^{2}=0.89 ; \theta: F(2.15,32.30)=71.00\right.$, $\left.\eta_{p}^{2}=0.83 ; \alpha: F(1.72,25.72)=9.94, \eta_{p}^{2}=0.40 ; \beta: F(1.29,19.37)=14.40, \eta_{p}^{2}=0.49\right)$, central $(\delta:$ $F(1.87,28.01)=468.45, \eta_{p}^{2}=0.97 ; \theta: F(2.00,30.01)=620.71, \eta_{p}^{2}=0.98 ; \alpha: F(3,45)=1001.63$, $\left.\eta_{p}^{2}=0.99 ; \beta: F(2.22,33.22)=364.97, \eta_{p}^{2}=0.96\right)$, motor $\left(\delta: F(1.77,26.59)=524.82, \eta_{p}^{2}=0.97\right.$ $\theta: F(1.76,26.36)=561.44, \quad \eta_{p}^{2}=0.97 ; \alpha: F(1.70,25.46)=808.81, \quad \eta_{p}^{2}=0.98 ; \beta:$ 
$\left.F(1.59,23.90)=623.88, \eta_{p}^{2}=0.98\right)$, parietal $\left(\delta: F(1.66,24.93)=938.08, \eta_{p}^{2}=0.98 ; \theta:\right.$

$F(1.92,28.84)=721.89 \quad, \quad \eta_{p}^{2}=0.98 \quad ; \quad \alpha \quad: \quad F(2.05,30.79)=649.08 \quad, \quad \eta_{p}^{2}=0.98 \quad ; \quad \beta \quad:$

$\left.F(1.56,23.41)=400.38, \eta_{p}^{2}=0.96\right)$, and occipital $\left(\delta: F(3,45)=587.78, \eta_{p}^{2}=0.98 ; \theta:\right.$

$F(1.85,27.69)=591.30, \eta_{p}^{2}=0.98 ; \alpha: F(1.96,29.37)=461.70, \eta_{p}^{2}=0.97 ; \beta: F(3,45)=329.34$, $\left.\eta_{p}^{2}=0.96\right)$ clusters

The post-hoc comparisons showed that delta band of the frontal $(p<0.001,|d|=0.61)$, central $(p<0.001,|d|=1.24)$, motor $(p<0.001,|d|=0.58)$, and parietal $(p<0.001,|d|=1.04)$ clusters and theta band of the central $(p<0.001,|d|=0.70)$ cluster significantly increased during the lanedeparture period relative to the baseline period. Conversely, significant event-related spectral suppressions were observed in the theta powers of the occipital cluster $(p<0.001,|d|=0.60)$, alpha powers of the motor $(p<0.001,|d|=2.46)$, parietal $(p<0.001,|d|=2.06)$, and occipital $(p<0.001$, $|d|=1.94)$ clusters, as well as beta powers of the central $(p<0.001,|d|=1.51)$, motor $(p<0.001$, $|d|=2.31)$, parietal $(p<0.001,|d|=2.12)$, and occipital $(p<0.001,|d|=2.08)$ clusters.

A significant spectral suppression was also observed during the steering period relative to the baseline period (post-hoc t-tests, FDR-adjusted $p$-value<0.001). In particular, this event-related desynchronization (ERD) occurred in the delta and theta bands of the frontal $(\delta: p<0.001,|d|=0.77$; $\theta: p<0.001,|d|=0.90)$, central $(\delta: p<0.001,|d|=1.11 ; \theta: p<0.001,|d|=1.71)$, motor $(\delta: p<0.001$, $|d|=2.09 ; \theta: p<0.001,|d|=2.89)$, parietal $(\delta: p<0.001,|d|=2.23 ; \theta: p<0.001,|d|=2.87)$, and occipital $(\delta: p<0.001,|d|=1.65 ; \theta: p<0.001,|d|=2.09)$ clusters and the alpha and beta bands of the central $(\alpha: p<0.001,|d|=2.95 ; \beta: p<0.001,|d|=1.39)$, motor $(\alpha: p<0.001,|d|=2.39 ; \beta$ : 
$p<0.001,|d|=1.55)$, parietal $(\alpha: p<0.001,|d|=2.39 ; \beta: p<0.001,|d|=1.40)$, and occipital $(\alpha$ : $p<0.001,|d|=1.93 ; \beta: p<0.001,|d|=1.13)$ clusters.

During the post-movement period, delta and theta powers of the central $(\delta: p<0.001$, $|d|=0.60 ; \theta: p<0.001,|d|=0.68)$ and parietal $(\delta: p<0.001,|d|=0.40, \theta: p<0.001,|d|=0.40)$ clusters were significantly less than that during the baseline period. Conversely, beta powers of the frontal $(p<0.001,|d|=0.75)$, central $(p<0.001,|d|=0.93)$, motor $(p<0.001,|d|=0.81)$, parietal $(p<0.001,|d|=0.83)$, and occipital $(p<0.001,|d|=1.27)$ clusters and alpha power of the occipital cluster $(p<0.001,|d|=0.54)$ during the post-movement period were significantly larger than that during the baseline period. Table 3 summarizes these results.

Main effect of performance quality

The main effect of the performance quality (three-way repeated measures ANOVA, FDRadjusted $p$-value<0.001)(Table 2) was observed in the frontal $\left(\delta: F(1.11,16.58)=81.43, \eta_{p}^{2}=0.84\right.$; $\theta: F(2,30)=85.37, \eta_{p}^{2}=0.85 ; \alpha: F(1.44,21.63)=74.16, \eta_{p}^{2}=0.83 ; \beta: F(1.43,21.47)=66.09$, $\left.\eta_{p}^{2}=0.82\right)$, central $\left(\delta: F(1.12,16.81)=141.32, \eta_{p}^{2}=0.90 ; \theta: F(1.12,16.81)=141.32, \eta_{p}^{2}=0.91\right.$ $\left.\alpha: F(2,30)=139.07, \eta_{p}^{2}=0.90 ; \beta: F(1.28,19.27)=224.96, \eta_{p}^{2}=0.94\right)$, motor $\quad(\delta:$ $F(1.46,21.86)=123.65, \eta_{p}^{2}=0.89 ; \theta: F(1.43,21.47)=106.54, \eta_{p}^{2}=0.88 ; \alpha: F(2,30)=78.90$, $\left.\eta_{p}^{2}=0.84 ; \beta: F(2,30)=91.17, \eta_{p}^{2}=0.86\right)$, parietal $\left(\delta: F(1.24,18.55)=151.15, \eta_{p}^{2}=0.91 ; \theta:\right.$ $F(1.38,20.68)=191.56, \eta_{p}^{2}=0.93 ; \alpha: F(2,30)=137.75, \eta_{p}^{2}=0.90 ; \beta: F(2,30)=301.52$, 
$\left.\eta_{p}^{2}=0.95\right)$, and occipital $\left(\delta: F(1.42,21.42)=454.03, \eta_{p}^{2}=0.97 ; \theta: F(2,30)=604.34, \eta_{p}^{2}=0.98\right.$;

$\left.\alpha: F(2,30)=155.00, \eta_{p}^{2}=0.91 ; \beta: F(2,30)=115.23, \eta_{p}^{2}=0.88\right)$ clusters.

The post-hoc comparisons showed that the power spectra of the suboptimal-performance group were significantly higher than those of the optimal-performance group in the delta band of the occipital cluster $(p<0.001,|d|=1.17)$ cluster, the theta band of the occipital $(p<0.001,|d|=1.29)$ cluster, the alpha band of the frontal $(p<0.001,|d|=0.73)$, parietal $(p<0.001,|d|=0.86)$, and occipital $(p<0.001,|d|=1.36)$ clusters, as well as the beta band of the central $(p<0.001,|d|=1.02)$, motor $(p<0.001,|d|=0.86)$, parietal $(p<0.001,|d|=1.17)$, and occipital $(p<0.001,|d|=1.37)$ clusters.

The post-hoc comparisons also indicated that all of the power spectra of the poor-performance group were significantly higher than those of the suboptimal-performance group in the frontal $(\delta$ : $p<0.001,|d|=1.63 ; \theta: p<0.001,|d|=1.56 ; \alpha: p<0.001,|d|=0.75 ; \beta: p<0.001,|d|=0.80)$, central $(\delta: p<0.001,|d|=1.38 ; \theta: p<0.001,|d|=1.52 ; \alpha: p<0.001,|d|=1.30 ; \beta: p<0.001,|d|=1.62)$, motor $(\delta: p<0.001,|d|=1.58 ; \theta: p<0.001,|d|=1.90 ; \alpha: p<0.001,|d|=0.85 ; \beta: p<0.001$, $|d|=0.55)$, parietal $(\delta: p<0.001,|d|=1.79 ; \theta: p<0.001,|d|=1.94 ; \alpha: p<0.001,|d|=0.93 ; \beta$ : $p<0.001,|d|=0.58)$, and occipital clusters $(\delta: p<0.001,|d|=2.55 ; \theta: p<0.001,|d|=2.42 ; \alpha$ : $p<0.001,|d|=0.94 ; \beta: p<0.001,|d|=0.54)$. All of the power spectra of the poor-performance group were also significantly greater than those of the optimal-performance group in all component clusters. Cumulatively, the power spectra revealed a transitive relation from optimal-, to suboptimal-, and poor-performance groups in the delta and theta bands of the occipital cluster, the 
alpha band of the frontal, parietal, and occipital clusters, as well as the beta band of the central, motor, parietal, and occipital clusters. The power spectra monotonically increased with a declining performance (i.e. optimal<suboptimal<poor). Table 4 summarizes these results.

Main effect of kinesthetic feedback

The main effect of the kinesthetic feedback (three-way repeated measures ANOVA, FDRadjusted $p$-value $<0.001)$ (Table 2 ) was observed in the delta band of the frontal $(F(1,15)=68.31$, $\left.\eta_{p}^{2}=0.82\right)$, central $\left(F(1,15)=93.65, \eta_{p}^{2}=0.86\right)$, motor $\left(F(1,15)=64.62, \eta_{p}^{2}=0.81\right)$, parietal $\left(F(1,15)=39.40, \eta_{p}^{2}=0.72\right)$, and occipital $\left(F(1,15)=50.69, \eta_{p}^{2}=0.77\right)$ clusters, the theta band of the frontal $\left(F(1,15)=102.10, \eta_{p}^{2}=0.87\right)$, central $\left(F(1,15)=168.67, \eta_{p}^{2}=0.92\right)$, and occipital $\left(F(1,15)=23.82, \eta_{p}^{2}=0.61\right)$ clusters, as well as the alpha band of the frontal $(F(1,15)=46.66$, $\left.\eta_{p}^{2}=0.76\right)$, central $\left(F(1,15)=85.16, \eta_{p}^{2}=0.85\right)$, and motor $\left(F(1,15)=30.37, \eta_{p}^{2}=0.67\right)$ clusters

The post-hoc comparisons further indicated that the power spectra in the delta band of the frontal $(p<0.001,|d|=0.39)$, central $(p<0.001,|d|=0.58)$, motor $(p<0.001,|d|=0.48)$, parietal $(p<0.001,|d|=0.58)$, and occipital $(p<0.001,|d|=0.60)$ clusters, the theta band of the frontal $(p<0.001,|d|=0.53)$, central $(p<0.001,|d|=0.67)$, and occipital $(p<0.001,|d|=0.37)$ clusters, and the alpha band of the frontal $(p<0.001,|d|=0.40)$, central $(p<0.001,|d|=0.64)$, and motor $(p<0.001,|d|=0.54)$ clusters under the $\mathrm{K}^{+}$condition were significantly less than that under the $\mathrm{K}^{-}$ condition. Table 5 summarizes these results. 


\subsection{Interaction Effects}

Event type by performance quality interaction

According to Table 2, the interaction effect of event type by performance quality (ExP) was significant (FDR-adjusted p-value $<0.001$ ) on the power spectra in four bands and five component clusters, except for the alpha power in the occipital cluster (Table 2). In particular, according to our results, a significant ExP interaction occurred in the theta band of the central cluster (Figs. 5A and 5C) $\left(F(3.92,58.81)=90.74, \quad \eta_{p}^{2}=0.87\right)$, the beta band of the motor cluster $\left(F(3.21,48.10)=79.62, \eta_{p}^{2}=0.86\right)$, the alpha band of the central cluster (Figs. 6A and 6C) $\left(F(2.94,44.08)=36.32, \eta_{p}^{2}=0.71\right)$, and the theta band of the frontal cluster (Figs. 7A and 7C) $\left(F(6,90)=17.46, \eta_{p}^{2}=0.54\right)$.

Post-hoc t-tests suggested that the event-related spectral perturbations might differ across different performance levels. During the lane-departure period, significant event-related synchronizations (ERS) of the delta and theta powers relative to the baseline spectra were found across most component clusters when optimal (frontal $\delta: p<0.001,|d|=1.91$; frontal $\theta: p<0.001$, $|d|=1.67$ (Fig. 7C); central $\delta: p<0.001,|d|=4.10$; central $\theta: p<0.001,|d|=3.23$ (Fig. 5C); motor $\delta: p<0.001,|d|=1.61 ;$ motor $\theta: p<0.001,|d|=0.72$; parietal $\delta: p<0.001,|d|=3.86$; parietal $\theta$ : $p<0.001,|d|=1.37$; occipital $\delta: p<0.001,|d|=0.98$ ) and suboptimal (frontal $\delta: p<0.001,|d|=1.98$; frontal $\theta: p<0.001,|d|=1.53$; central $\delta: p<0.001,|d|=4.10$, central $\theta: p<0.001,|d|=1.22$; motor $\delta$ : $p<0.001,|d|=0.85$; parietal $\delta: p<0.001,|d|=2.22$ ) performances were achieved. However, neither delta-ERS nor theta-ERS was significant $(p>0.001)$ in the poor-performance state. Conversely, the 
theta powers of motor, parietal and occipital clusters showed a significant ERD when the performance was suboptimal (motor: $p<0.001,|d|=1.03$; occipital: $p<0.001,|d|=1.15$ ) and poor (motor: $p<0.001,|d|=1.55$; parietal: $p<0.001,|d|=1.38$; occipital: $p<0.001,|d|=1.02$ ). For alpha and beta bands, significant ERDs were observed,mainly across most component clusters except for the frontal cluster during the lane-departure period regardless of the changes in the performance state.

During the steering period, significant delta-, theta-, alpha-, and beta-ERDs were found in most component clusters in the optimal- (frontal $\alpha: p<0.001,|d|=1.00$; frontal $\beta: p<0.001,|d|=1.54$; central $\theta: p<0.001,|d|=1.60$; central $\alpha: p<0.001,|d|=3.17$; central $\beta: p<0.001,|d|=4.21$; motor $\delta: p<0.001,|d|=1.65$; motor $\theta: p<0.001,|d|=2.07$; motor $\alpha: p<0.001,|d|=4.25$; motor $\beta$ : $p<0.001,|d|=5.80 ;$ parietal $\delta: p<0.001,|d|=2.05$; parietal $\theta: p<0.001,|d|=2.23 ;$ parietal $\alpha$ : $p<0.001,|d|=2.91 ;$ parietal $\beta: p<0.001,|d|=4.15 ;$ occipital $\delta: p<0.001,|d|=1.22 ;$ occipital $\theta$ : $p<0.001,|d|=1.90$; occipital $\alpha: p<0.001,|d|=2.56$; occipital $\beta: p<0.001,|d|=2.98)$, suboptimal(frontal $\delta: p<0.001,|d|=1.24$; frontal $\theta: p<0.001,|d|=1.39$; frontal $\alpha: p<0.001,|d|=1.61$; frontal $\beta: p<0.001,|d|=1.60 ;$ central $\delta: p<0.001,|d|=1.72$; central $\theta: p<0.001,|d|=2.98$; central $\alpha$ : $p<0.001,|d|=5.04$; central $\beta: p<0.001,|d|=4.22$; motor $\delta: p<0.001,|d|=3.35$; motor $\theta: p<0.001$, $|d|=4.91$; motor $\alpha: p<0.001,|d|=4.78$; motor $\beta: p<0.001,|d|=5.49$; parietal $\delta: p<0.001$, $|d|=3.54$; parietal $\theta: p<0.001,|d|=4.76$; parietal $\alpha: p<0.001,|d|=6.37$; parietal $\beta: p<0.001$, $|d|=5.60$; occipital $\delta: p<0.001,|d|=2.31$; occipital $\theta: p<0.001,|d|=3.22$; occipital $\alpha: p<0.001$, $|d|=4.06$; occipital $\beta: p<0.001,|d|=2.93$ ), and poor-performance (frontal $\delta: p<0.001,|d|=1.14$; 
frontal $\theta: p<0.001,|d|=0.87$; central $\delta: p<0.001,|d|=4.63$; central $\theta: p<0.001,|d|=5.13$; central $\alpha: p<0.001,|d|=2.63$; motor $\delta: p<0.001,|d|=3.17$; motor $\theta: p<0.001,|d|=3.18$; motor $\alpha$ : $p<0.001,|d|=1.88$; parietal $\delta: p<0.001,|d|=4.55$; parietal $\theta: p<0.001,|d|=4.73$; parietal $\alpha$ : $p<0.001,|d|=2.58$; occipital $\delta: p<0.001,|d|=2.37$; occipital $\theta: p<0.001,|d|=2.14$; occipital $\alpha$ : $p<0.001,|d|=1.20)$ states.

During the post-movement period, delta- and theta-ERDs were found across most component clusters yet only in the poor-performance state (frontal $\delta: p<0.001,|d|=0.81$; central $\delta$ : $p<0.001$, $|d|=1.62$; central $\theta: p<0.001,|d|=1.56$; motor $\delta: p<0.001,|d|=0.87$; parietal $\delta: p<0.001$, $|d|=1.33$; parietal $\theta: p<0.001,|d|=0.83$; occipital $\delta: p<0.001,|d|=0.81$; occipital $\theta: p<0.001$, $|d|=0.79)$. Conversely, significant augments of the beta power were found across optimal(parietal: $p<0.001,|d|=0.67$; occipital: $p<0.001,|d|=1.57$ ), suboptimal- (frontal: $p<0.001$, $|d|=0.86$; central: $p<0.001,|d|=1.06$; occipital: $p<0.001,|d|=1.09$ ), and poor-performance (frontal: $p<0.001,|d|=1.70$; central: $p<0.001,|d|=1.94$; motor: $p<0.001,|d|=2.60$; parietal: $p<0.001,|d|=1.97$; occipital: $p<0.001,|d|=1.55)$ states. Some spectral augments were also observed in the delta, theta, and alpha powers of the occipital cluster $(\delta: p<0.001,|d|=0.89 ; \theta$ : $p<0.001,|d|=1.01 ; \alpha: p<0.001,|d|=1.36)$ in the optimal-performance state and the alpha powers of the frontal $(p<0.001,|d|=0.80)$, motor $(p<0.001,|d|=1.24)$, and parietal $(p<0.001,|d|=0.66)$ clusters in the poor-performance state. Table 6 summarizes these results.

The post-hoc t-testes indicated that regardless of the event types, the delta and theta powers of the occipital cluster monotonically increased (i.e. poor>suboptimal>optimal) with a declining 
performance. In particular, significantly larger delta and theta powers of the occipital cluster were found in the suboptimal-performance state than those in the optimal-performance state during the baseline $(\delta: p<0.001,|d|=1.83 ; \theta: p<0.001,|d|=2.09)$, lane-departure $(\delta: p<0.001,|d|=0.82 ; \theta$ : $p<0.001,|d|=1.11)$, steering $(\delta: p<0.001,|d|=0.70 ; \theta: p<0.001,|d|=0.72)$, and post-movement $(\delta: p<0.001,|d|=2.26 ; \theta: p<0.001,|d|=2.06)$ periods. Moreover, significantly larger delta and theta powers of the occipital cluster were found in the poor-performance state than those in the suboptimal-performance state during the baseline $(\delta: p<0.001,|d|=4.42 ; \theta: p<0.001,|d|=4.29)$, lane-departure $(\delta: p<0.001,|d|=2.99 ; \theta: p<0.001,|d|=3.57)$, steering $(\delta: p<0.001,|d|=2.64 ; \theta$ : $p<0.001,|d|=2.92)$, and post-movement $(\delta: p<0.001,|d|=4.33 ; \theta: p<0.001,|d|=3.84)$ periods. However, the post-hoc comparisons also suggested that the performance-related spectral changes might vary across different event types. For instance, with a declining performance, the delta and theta powers of all component clusters monotonically increased only during the baseline and postmovement periods. In particular, during the baseline period, the delta and theta powers of the frontal (Fig. 7A) and central clusters (Fig. 5A) in suboptimal- and poor-performance states were significantly larger than those in the optimal- (frontal $\delta: p<0.001,|d|=0.91$; frontal $\theta: p<0.001$, $|d|=1.10$; central $\delta: p<0.001,|d|=0.85$; central $\theta: p<0.001,|d|=1.05$ ) and suboptimalperformance (frontal $\delta: p<0.001,|d|=2.16$; frontal $\theta: p<0.001,|d|=2.42$; central $\delta: p<0.001$, $|d|=3.88$; central $\theta: p<0.001,|d|=3.06$ ) states, respectively. During the post-movement period, the delta and theta powers of the frontal (Fig. 7A) and central clusters (Fig. 5A) in the suboptimaland poor-performance states were significantly larger than that in the optimal- (frontal $\delta: p<0.001$, 
$|d|=1.04$; frontal $\theta: p<0.001,|d|=1.28$; central $\delta: p<0.001,|d|=0.90$; central $\theta: p<0.001$,

$|d|=1.18$ ) and suboptimal-performance (frontal $\delta: p<0.001,|d|=2.02$; frontal $\theta: p<0.001$, $|d|=2.13$; central $\delta: p<0.001,|d|=2.88$; central $\theta: p<0.001,|d|=2.87$ ) states, respectively.

However, delta and theta powers of the frontal, central, motor, parietal, and occipital cluster in suboptimal- and poor-performance states were insignificantly larger than those in the optimal- and suboptimal-performance states ( $p>0.001)$. Table 7 summarizes these results.

Event type by kinesthetic feedback interaction

According to Table 2, the interaction effect of event type by kinesthetic feedback $(\mathrm{E} \times \mathrm{K})$ was significant (FDR-adjusted $p$-value $<0.001)$ on the power spectra of the frontal $(\delta: F(3,45)=17.45$, $\eta_{p}^{2}=0.54 ; \theta: F(2.72,40.75)=11.19, \eta_{p}^{2}=0.43 ; \alpha: F(3,45)=11.00, \eta_{p}^{2}=0.42 ; \beta:$ $\left.F(3,45)=7.95, \eta_{p}^{2}=0.35\right)$, central $\left(\theta: F(2.03,30.46)=24.39, \eta_{p}^{2}=0.62 ; \alpha: F(3,45)=90.53\right.$, $\left.\eta_{p}^{2}=0.86 ; \beta: F(3,45)=17.13, \eta_{p}^{2}=0.53\right)$, motor $\left(\delta: F(3,45)=14.02, \eta_{p}^{2}=0.48 ; \theta:\right.$ $F(3,45)=19.35, \eta_{p}^{2}=0.56 ; \alpha: F(1.98,29.65)=14.71, \eta_{p}^{2}=0.50 ; \beta: F(3,45)=12.05$, $\left.\eta_{p}^{2}=0.45\right)$, parietal $\left(\delta: F(3,45)=8.15, \eta_{p}^{2}=0.35 ; \theta: F(3,45)=11.05, \eta_{p}^{2}=0.42 ; \alpha:\right.$ $\left.F(3,45)=16.75, \eta_{p}^{2}=0.53\right)$, and occipital $\left(\delta: F(2.17,32.47)=21.77, \eta_{p}^{2}=0.59 ; \theta:\right.$ $\left.F(3,45)=25.79, \eta_{p}^{2}=0.63 ; \alpha: F(3,45)=33.80, \eta_{p}^{2}=0.69 ; \beta: F(3,45)=12.51, \eta_{p}^{2}=0.45\right)$ clusters, except for the delta power of the central cluster and the beta power of the parietal cluster $(p>0.001)$ 
Post-hoc t-tests suggested that the $\mathrm{K}^{-}$and $\mathrm{K}^{+}$sessions might differ in the event-related spectral perturbations. During the lane-departure period, delta $(p<0.001,|d|=0.80)$ and theta $(p<0.001$, $|d|=0.54)$ powers of the frontal cluster (Fig. 7E), theta power of the central cluster $(p<0.001$, $|d|=0.99)($ Fig. 5E), as well as delta power of the motor cluster $(p<0.001,|d|=0.86)$ significantly increased only in the $\mathrm{K}^{-}$session. Conversely, the beta power of the frontal cluster $(p<0.001$, $|d|=0.69)$, alpha power of the central cluster $(p<0.001,|d|=1.36)$ (Fig. 6E), theta powers of motor $(p<0.001,|d|=0.67)$ and occipital clusters $(p<0.001,|d|=0.86)$ significantly decreased only in the $\mathrm{K}^{+}$session. During the steering period, the theta (Fig. 7E) and alpha powers of the frontal cluster were significantly suppressed $(\theta: p<0.001,|d|=1.20 ; \quad \alpha: p<0.001,|d|=0.78)$ only in the $\mathrm{K}^{+}$ session. During the post-movement period, alpha power of the occipital cluster significantly increased only in the $\mathrm{K}^{+}$session. Table 8 summarizes these results.

Post-hoc t-tests also suggested that the power spectra of the $\mathrm{K}^{-}$and $\mathrm{K}^{+}$sessions might differ across different event types, except for the baseline. During the lane-departure period, power spectra of the frontal $(\alpha: p<0.001,|d|=0.73)$, central $(\theta: p<0.001,|d|=1.32 ; \alpha: p<0.001$, $|d|=1.80)$, motor $(\delta: p<0.001,|d|=0.73 ; \theta: p<0.001,|d|=0.79 ; \alpha: p<0.001,|d|=0.98)$, parietal clusters $(\delta: p<0.001,|d|=0.67 ; \theta: p<0.001,|d|=0.67)$ in the $\mathrm{K}^{+}$session were significantly less than those in the $\mathrm{K}^{-}$session. During the steering period, the power spectra of the frontal $(\delta: p<0.001$, $|d|=0.87 ; \theta: p<0.001,|d|=0.86 ; \alpha: p<0.001,|d|=0.62)$, central $(\theta: p<0.001,|d|=1.07 ; \alpha$ : $p<0.001,|d|=1.33)$, motor $(\delta: p<0.001,|d|=0.82 ; \theta: p<0.001,|d|=0.59 ; \alpha: p<0.001,|d|=0.86)$, parietal $(\delta: p<0.001,|d|=0.87 ; \theta: p<0.001,|d|=0.65 ;)$ and occipital clusters $(\delta: p<0.001$, 
$|d|=1.66 ; \theta: p<0.001,|d|=1.30 ; \alpha: p<0.001,|d|=1.05)$ in the $\mathrm{K}^{+}$session were significantly less than those in the $\mathrm{K}^{-}$session. During the post-movement period, the power spectra of the parietal ( $\alpha$ : $p<0.001,|d|=0.96)$ and occipital clusters $(\beta: p<0.001,|d|=0.62)$ in the $\mathrm{K}^{+}$session were significantly larger than those in the $\mathrm{K}^{-}$session. Table 9 summarizes these results.

Performance quality by kinesthetic feedback interaction

According to Table 2, the interaction effect of performance quality by kinesthetic feedback $(\mathrm{P} \times \mathrm{K})$ was significant $($ FDR-adjusted p-value $<0.001)$ on theta, alpha, and beta powers of the frontal cluster $\left(\theta: F(1.61,24.19)=13.88, \eta_{p}^{2}=0.48, \alpha: F(2,30)=16.57, \eta_{p}^{2}=0.48, \beta:\right.$ $\left.F(1.38,20.67)=16.49, \eta_{p}^{2}=0.48\right)$, delta power of the parietal cluster $(F(2,30)=10.14$, $\left.\eta_{p}^{2}=0.40\right)$, as well as delta power of the occipital clusters $\left(F(2,30)=10.00, \eta_{p}^{2}=0.40\right)$

Most of the performance-related spectral changes in the $\mathrm{K}^{+}$session showed a transitive relation between performance-groups that was the same as in the $\mathrm{K}^{-}$session. However, post-hoc t-tests suggested that the $\mathrm{K}^{-}$and $\mathrm{K}^{+}$sessions differed in performance-related spectral changes. Beta power of the frontal cluster ( $\mathrm{K}^{-}$session), delta and theta powers of the central cluster ( $\mathrm{K}^{-}$session), delta and theta powers of the motor cluster $\left(\mathrm{K}^{-}\right.$session), and alpha power of the motor cluster $\left(\mathrm{K}^{+}\right.$session) did not significantly increase $(p>0.001)$ with a declining performance from optimal to suboptimal. Additionally, beta power of the occipital cluster did not significantly increase $(p>0.001)$ in the $\mathrm{K}^{+}$ session with a declining performance from suboptimal to poor. Table 10 summarizes these results. 
In the optimal-performance state, the $\mathrm{K}^{-}$session and the $\mathrm{K}^{+}$session did not statistically differ significantly in all power spectra of the frontal, parietal, and occipital clusters $(p>0.001)$. Conversely, the power spectra of the central $(\delta: p<0.001,|d|=1.30 ; \theta: p<0.001,|d|=1.12 ; \alpha$ : $p<0.001,|d|=0.69)$ and motor $(\delta: p<0.001,|d|=0.96 ; \theta: p<0.001,|d|=0.49)$ clusters in the $\mathrm{K}^{-}$ session were significantly larger than those in the $\mathrm{K}^{+}$session. In the suboptimal-performance state, theta power of the frontal cluster $(p<0.001,|d|=0.52)$ and alpha powers of the central $(p<0.001$, $|d|=0.48)$ and motor $(p<0.001,|d|=0.81)$ clusters in the $\mathrm{K}^{-}$session were significantly larger than that in the $\mathrm{K}^{+}$session. Conversely, alpha power of the parietal cluster $(p<0.001,|d|=0.53)$ and beta powers of the frontal $(p<0.001,|d|=0.54)$, central $(p<0.001,|d|=0.53)$, parietal $(p<0.001$, $|d|=0.56)$, and occipital $(p<0.001,|d|=0.54)$ clusters in the $\mathrm{K}^{-}$session were significantly less than those in the $\mathrm{K}^{+}$session. In the poor-performance state, most power spectra of the frontal $(\delta$ : $p<0.001,|d|=0.67 ; \theta: p<0.001,|d|=0.88 ; \alpha: p<0.001,|d|=0.93 ; \beta: p<0.001,|d|=0.79)$, central $(\delta: p<0.001,|d|=0.62 ; \theta: p<0.001,|d|=0.75 ; \alpha: p<0.001,|d|=0.76)$, motor $(\delta: p<0.001$, $|d|=0.59 ; \alpha: p<0.001,|d|=0.75 ; \beta: p<0.001,|d|=0.51)$, parietal $(\delta: p<0.001,|d|=1.21 ; \theta$ : $p<0.001,|d|=0.77)$, and occipital $(\delta: p<0.001,|d|=1.35 ; \theta: p<0.001,|d|=0.95)$ clusters in the $\mathrm{K}^{-}$ session were significantly larger than those in the $\mathrm{K}^{+}$session. Cumulatively, in all performance levels, post-hoc t-tests indicated that two sessions in the alpha band of the occipital cluster statistically differ significantly. Additionally, alpha power of the central cluster in the $\mathrm{K}^{-}$session was significantly larger than that in the $\mathrm{K}^{+}$session across all performance levels. Table 11 summarizes these results. 
Three-way interaction

According to Table 2, the three-way interaction effect of $\mathrm{E} \times \mathrm{P} \times \mathrm{K}$ was significant (FDRadjusted p-value $<0.001)$ on delta activities of the frontal $\left(F(6,90)=7.03, \eta_{p}^{2}=0.32\right)$ and motor clusters $\left(F(6,90)=4.54, \eta_{p}^{2}=0.23\right)$, alpha activity of the central cluster $(F(6,90)=9.74$, $\left.\eta_{p}^{2}=0.39\right)$, as well as beta activity of the occipital cluster $\left(F(6,90)=7.17, \eta_{p}^{2}=0.32\right)$. The following post-hoc comparisons were made to determine whether two-way interactions vary across levels of a third variable.

For delta activity of the frontal cluster, all of the two-way interactions varied across levels of the third variable. Specifically, the interactions effects of ExP differed across various levels of kinesthetic feedback $\left(\mathrm{K}^{+}\right.$session: $p<0.001, \quad F(6,90)=22.27 ; \mathrm{K}^{-} \quad$ session: $p>0.001$, $F(6,90)=3.12)$. The interaction effect of $\mathrm{E} \times \mathrm{K}$ differed across various levels of performance quality (optimal: $p>0.001, F(3,45)=0.56$; suboptimal: $p>0.001, F(3,45)=5.89$; poor: $p<0.001$, $F(3,45)=14.59$ ). Additionally, the interaction effect of $\mathrm{P} \times \mathrm{K}$ differed across various event types (baseline: $p>0.001, F(2,30)=0.98$; lane-departure: $p>0.001, F(2,30)=1.47$; steering: $p<0.001$, $F(2,30)=23.33$; post-movement: $p<0.001, F(2,30)=12.36)$.

For delta power of the motor cluster, all of the interactions effects of ExP were statistically significant across various levels of kinesthetic feedback $\left(\mathrm{K}^{+}\right.$session: $p<0.001, F(6,90)=21.58 ; \mathrm{K}^{-}$ session: $p<0.001, F(6,90)=18.91)$. The interaction effect of E×K differed across various levels of performance quality (optimal: $p>0.001, F(3,45)=6.46$; suboptimal: $p>0.001, F(3,45)=5.30$; 
poor: $p<0.001, F(3,45)=15.54)$. Additionally, the interaction effect of $\mathrm{P} \times \mathrm{K}$ differed across various event types (baseline: $p>0.001, F(2,30)=2.15$; lane-departure: $p>0.001, F(2,30)=8.08$; steering: $p>0.001, F(2,30)=6.32$; post-movement: $p<0.001, F(2,30)=10.87)$.

For alpha activity of the central cluster, all of the interactions effects of ExP were statistically significant across various levels of kinesthetic feedback $\left(\mathrm{K}^{+}\right.$session: $p<0.001, F(6,90)=17.58 ; \mathrm{K}^{-}$ session: $p<0.001, F(6,90)=31.80)$. All of the interactions effects of $\mathrm{E} \times \mathrm{K}$ were also statistically significant across various levels of performance quality (optimal: $p<0.001, F(3,45)=41.64$; suboptimal: $p<0.001, F(3,45)=95.10$; poor: $p<0.001, F(3,45)=15.22)$. Conversely, the interaction effect of $\mathrm{P} \times \mathrm{K}$ differed across various event types (baseline: $p>0.001, F(2,30)=1.26$; lane-departure: $p>0.001, F(2,30)=6.79$; steering: $p<0.001, F(2,30)=9.04$; post-movement: $p>0.001, F(2,30)=3.56)$

For beta activity of the occipital cluster, all of the interactions effects of ExP were statistically significant across various levels of kinesthetic feedback $\left(\mathrm{K}^{+}\right.$session: $p<0.001$, $F(2.22,33.34)=15.23 ; \mathrm{K}^{-}$session: $\left.p<0.001, F(6,90)=5.93\right)$. Additionally, all of the interaction effects of $\mathrm{P} \times \mathrm{K}$ were not statistically significant across various event types (baseline: $p>0.001$, $F(2,30)=7.77$; lane-departure: $p>0.001, F(2,30)=6.19 ;$ steering: $p>0.001, F(2,30)=0.45$; post-movement: $p>0.001, F(2,30)=0.32)$. Additionally, the interaction effect of ExK differed across various levels of performance quality (optimal: $p>0.001, F(3,45)=1.97$; suboptimal: $p<0.001, F(3,45)=16.79$; poor: $p<0.001, F(3,45)=11.54)$. 


\section{Discussion}

This study has thoroughly elucidated how performance lapse and kinesthetic feedback collaboratively affect brain activity during a sustained-attention driving task using independent component analysis, time-frequency analysis, and three-way repeated measure ANOVA. Empirical results demonstrated the neural dynamics of multisensory integration in a natural environment. Consistent with previous studies (Banks et al., 2004; Boyle et al., 2008; Campagne et al., 2004; Eoh et al., 2005; Huang et al., 2009; Jap et al., 2009; Otmani et al., 2005), the results of our statistical analysis (Table 2) indicated that EEG dynamics in all spectral bands and component clusters were significantly altered following behavioral lapses. Additionally, most of the spectral bands and component clusters were highly correlated with the changes of RT (Fig. 4), indicating a transitive relation from optimal-, to suboptimal, and poor (i.e., optimal<suboptimal<poor), especially in the $\mathrm{K}^{+}$sessions (Table 10). The driving task involved lane-departure events and steering activities that induced significant ERS and ERD, respectively. The theta-ERS was found during the lane-departure period in multiple component clusters, particularly in optimal- and suboptimal-performance states (Table 6). Our results further demonstrated that less theta-ERS occurred in the frontal and central clusters in the $\mathrm{K}^{+}$session than that in the $\mathrm{K}^{-}$session (Tables 8 and 9). For most of the spectra bands and component clusters, ERD occurred during the steering period across all performance levels (Table 6). These results indicated that, across all performance levels, less alpha power of the central cluster was observed in the $\mathrm{K}^{+}$session than that in the $\mathrm{K}^{-}$ session (Table 11). Cumulatively, when individuals performed the driving task involving 
kinesthetic feedback, less EEG activity in delta, theta, and alpha bands was observed in multiple component clusters (Table 5).

Comparing the RTs in the $\mathrm{K}^{-}$session with those in the $\mathrm{K}^{+}$session (Kolmogorov-Smirnov test, $p<0.001$ ) showed that subjects performed better (i.e., a faster reaction time) in the driving task when they had kinesthetic feedback to integrate with incoming visual information. However, the kinesthetic feedback appeared to be beneficial only when the subjects were in the optimalperformance state. Their reaction time tended to be slow under the $\mathrm{K}^{+}$condition, compared to the $\mathrm{K}^{-}$condition, when the subjects were in a suboptimal-performance state.

\subsection{Kinesthesia modifies tonic EEG dynamics}

Neural mechanisms mediate lapse-related cortical activity

Results of correlation between the power spectra and the RT showed that the synchronized changes of EEG activities in all spectral bands are comparable between frontal, central, motor, parietal and occipital clusters, which might reflect a signal from the deep brain projecting to these cortical areas (Chuang et al., 2012). The cholinergic neurons in the basal forebrain projecting much of the remaining cerebral cortices might be a driver for maintenance of behavioral performance. Thalamus is also one of the key lapse-related regions (Chee et al., 2008). Studies have reported that thalamo-cortical networks strongly affect the spectral synchronization of cortical field potentials (Herculano-Houzel et al., 1999). This co-variation of EEG spectral fluctuations may be caused by the reduction of cortical-cortical connectivity (Massimini et al., 2005) which produces a cortical 
gate during the transition from optimal to poor performance. The cortical gate acts like a low-pass filter, allowing slow, widespread activity to propagate through the brain, while blocking the propagation of fast fluctuations (Esser et al., 2009).

Power spectral augmentations across most independent brain processes

Many studies (Davidson et al., 2007; Lin et al., 2010; Makeig and Inlow, 1993; Peiris et al., 2006) have investigated behavioral lapses by using EEG measurements. According to a metaanalysis (Lin et al., 2012a), most EEG activities in low bands identified significant increases at most of brain regions, which were strongly correlated with the deterioration of task performance. However, this study demonstrated that in a static (laboratory) environment, the differences of delta and theta powers in the central and motor clusters between the optimal- and suboptimalperformance groups were insignificant (Table 10). Conversely, in a dynamic environment, kinesthetic inputs induced decreased EEG activities in the delta and theta bands, particularly in the optimal-performance state (Table 11), resulting in a transitive relationship of power spectra from optimal-, to suboptimal-, and poor-performance groups across most component clusters (Table 10). This finding suggests that the EEG spectral dynamics are sensitive to performance lapses when involved with the kinesthetic feedback. Hence, for real-world applications, such as performance lapse prediction/detection, developing an advanced algorithm is necessary to adaptive to the environment. 


\subsection{Kinesthesia modifies phasic EEG dynamics}

Reduced theta-ERS at central and frontal clusters

The event-related theta activity typically is associated with the level of cognitive demand (Klimesch, 1999; Onton et al., 2005; Rawle et al., 2012; Sauseng et al., 2007) and the amount of attentional resources (Gomarus et al., 2006) for planning the required motor action (Perfetti et al., 2011) and integrating the sensorimotor function (Cruikshank et al., 2012). A parallel increase of fronto-parietal theta coherence mirrors activation of a fronto-parietal network for central executive functions of working memory (Sauseng et al., 2005). The human brain is highly responsive to movements of the platform and, therefore, to somatic sensation. The difference of power spectra between the $\mathrm{K}^{-}$and $\mathrm{K}^{+}$sessions may arise from the different amounts of attentional resources for engaging in the driving task. When the environment provided only visual input, the subject had to pay more attention to the trajectory of the vehicle to detect a random lane-departure event. Kinesthetic feedback might provide critical input that facilitated the driving task such that the subjects did not have to focus on the vehicle trajectory at all times.

Furthermore, the dipole model of the frontal component cluster was located near the medial frontal area involving the cingulate cortex. The anterior cingulate cortex has been identified to be associated with the error processing (Calhoun and Pearlson, 2012; van Veen and Carter, 2002). Results of the event-related fMRI experiments (Holroyd et al., 2004) also illustrated that an error feedback as well as an error response would activate the region of dorsal anterior cingulate cortex. In previous EEG studies, the theta activity dominates the error-related negativity which is 
considered as an indicator of brain processes to monitor action outcomes (Luu et al., 2004). Our results (Table 9 and Fig. 7E) demonstrate that the kinesthetic feedback from the moving vehicle significantly reduces theta augmentation following the onset of deviation (during the lane-departure period) below that in the $\mathrm{K}^{-}$session. This result may suggest that an external source of the error information will facilitate the brain resource for error monitoring.

Event-related alpha desynchronization in the central cluster

Control of the steering wheel causes an observable decrease in the alpha power that dominates most regions of the brain (Table and Fig. 4). An apparent ERD of alpha power shortly after the onset of the deviation reflects the needs for shifting cortical activity from an idle state to an activated state (Goldman et al., 2002). Accordingly, a power suppression of 2-4 dB relative to the baseline level is required to move the steering wheel to respond to the perturbation, and a strongly positive relationship between the RT and the alpha power provides evidence that greater alpha suppression is associated with a more timely response (Perfetti et al., 2011; Zhang et al., 2008). Consistent with a previous study (Cochin et al., 1998), this study found that the decrease in alphaband power in the $\mathrm{K}^{+}$session exceeded that in the $\mathrm{K}^{-}$session in the central cluster (Table 9 and Figs. $6 \mathrm{~B}$ and $6 \mathrm{E}$ ). This result suggests that kinesthesia can produce large cortical excitability (Klimesch et al., 2007) as part of an action response. 


\subsection{Driving performance}

Upon a perturbation, the attempt to minimize the trajectory error generates a positively skewed distribution of reaction times (Fig. 3), which was truncated on (Lal and Craig, 2001) the left and spread out on the right (Triggs and Harris, 1982). In this study, an RT of 0.756s was used as the upper bound on the optimal-performance group. This threshold is close to the RT values of $700 \mathrm{~ms}$ and $750 \mathrm{~ms}$, which are suggested by the Department of Transport in Britain and the Royal Automobile Club of Victoria, Australia, respectively, for calculating safe tailing distances and stopping distances (Nguyen et al., 2000). Double the threshold for the optimal- performance group (with an RT of 1.512s), was set as the lower bound on the poor-performance group. Notably, the vehicle would hit the curb of the roadside within $1.5 \mathrm{~s}$ of the onset of a deviation event. Subjects with an RT over this threshold indicated that they were unaware of the lane-departure event and the vehicle eventually hit the roadside.

The effective integration of multiple sensory modalities from the body and the environment enable the driver to understand the surrounding feedback and accurately respond to the traffic event. The established immersive driving environment (Fig. 1), including the surroundings and motion platform reproduces two sensory modalities - visual and kinesthetic input - that inform the subject. Consistent with a previous finding (Ngo et al., 2012), multisensory feedback could effectively alert drivers to take corrective action. Restated, the involvement of kinesthetic feedback facilitates a fast RT in response to an unintended lane departure. However, the kinesthetic feedback appeared to be beneficial only when the performance was optimal. Comparing the two conditions with respect to 
the median RT of the suboptimal-performance group between two conditions showed that the reaction time of a subject tended to be slow when driving under the $\mathrm{K}^{+}$condition. Notably, a valid concern arises that facilitating the kinesthetic feedback on a driving task, as reflected by the nonsignificant theta-ERS of the central component during lane departure in the $\mathrm{K}^{+}$session (Table 8), may reduce situational awareness (Young and Stanton, 2007) and lower task engagement (Neubauer et al., 2012).

\subsection{Limitations}

This study implemented an event-related lane-departure driving paradigm on a dynamic simulator to explore the detailed EEG dynamics associated with kinesthetic feedback under different levels of task performance, as measured by the RT to a random lane-departure event. However, the lack of independent indicators to measure the subject's level of arousal makes it difficult to identify the cause of a long RT. Given this limitation, the EEG dynamics of this study must be interpreted in terms of behavioral lapses. Additionally, varying levels of sleep in participants can lead to various levels of homeostatic changes in the brain, thus confounding the EEG dynamics. To ensure rigorous monitoring of sleep habits, we recommend using a sleep diary or ActiWatch (Lotjonen et al., 2003) for at least a week before the study.

\section{Conclusion}


This study thoroughly elucidated the brain dynamics and behavioral changes of a driver who responded to a lane-departure event in a static or dynamic driving simulator. Independent component analysis was performed to decompose 30-channel EEG data into spatially independent brain processes. Independent components with similar topographic maps, dipole source locations, and spectral profiles were then grouped into IC clusters. Tonic analysis demonstrates that the increasing activities in multiple bands and brain regions are robust indices of behavioral lapses, especially when driving involves kinesthetic feedback. Moreover, a decrease in phasic theta augmentation in the central component suggests that the motion cues facilitate the distribution of attentional resources. Whereas an increase in phasic alpha suppression in the central cluster suggests that cortical excitability increased in the presence of motion cues. Although kinesthetic feedback can improve driving performance in terms of reaction time, a decrease in attentional demand (as facilitated by kinesthetic feedback) may increase the reaction time in the suboptimalperformance state. Results of this study demonstrate the importance of kinesthetic feedback in driving from an EEG dynamics perspective. Neurophysiological evidence of brain-behavior relations in this study provides further insight into distributed brain dynamics that support natural human cognition and complex coordinated, multi-joint naturalistic behavior, ultimately shedding light on the characteristics of brain-behavior relations in operational environments. 


\section{Acknowledgements}

The authors would like to express their sincere appreciation and gratitude to anonymous reviewers for their thorough review and invaluable comments, which contributed substantially toward improving the quality of this article. This work was supported in part by the UST-UCSD International Center of Excellence in Advanced Bio-engineering sponsored by the Taiwan National Science Council I-RiCE Program under Grant Number: NSC-101-2911-I-009-101, in part by the Aiming for the Top University Plan of National Chiao Tung University, the Ministry of Education, Taiwan, under Contract 100W9633. Research was also sponsored in part by the Army Research Laboratory and was accomplished under Cooperative Agreement Number W911NF-10-2-0022. The views and the conclusions contained in this document are those of the authors and should not be interpreted as representing the official policies, either expressed or implied, of the Army Research Laboratory or the U.S Government. The U.S Government is authorized to reproduce and distribute reprints for Government purposes notwithstanding any copyright notation herein. Ted Knoy is appreciated for his editorial assistance. 


\section{Figure legends}

\section{Figure 1.}

(A) Immersive driving environment, including a real car frame that is mounted on a Stewart platform and a projected night-time driving scene (Lin et al., 2010).

(B) Event-related lane departure paradigm (Huang et al., 2009). Onset of deviation occurs when the vehicle begins to deviate. Onset of response occurs when the subject initiates compensatory steering. End of response occurs when the vehicle returns to the center of the cruising lane and the subject ceases to rotate the steering wheel.

\section{Figure 2.}

(A) Average scalp topographies of frontal, central, motor, parietal, and occipital clusters, estimated from all ICs of interest.

(B) Equivalent dipole locations and their averages. The average Talairach coordinates of the frontal, central, motor, parietal, and occipital components are $(-2.2,30.3,22.3),(0.7,-2.3,40.0),(-31.0$, $26.5,-41.4),(2.0,-41.8,32.9)$, and $( \pm 3.7,-71.5,4.4)$, respectively.

\section{Figure 3.}

(A) Histograms of the latency of the onset of response (reddish distribution), end of response (greenish distribution), and the onset of the next deviation (bluish distribution) observed in the $\mathrm{K}^{-}$ session.

(B) Histograms of the latency of the onset of response (red distribution), end of response (green distribution), and the onset of the next deviation (blue distribution) observed in the $\mathrm{K}^{+}$session.

(C) The times of occurrence of the onset of the deviation (dashed line), the onset of the response (dashed trace), the end of the response (dotted-dashed trace), and the onset of the next deviation (solid trace), which were sorted by RT and vertically stacked. Color traces and black lines represent the times of the occurrence of the events and their median times, respectively.

\section{Figure 4.}

Tonic and phasic EEG dynamics in (A) frontal, (B) central, (C) motor, (D) parietal, and (E) occipital clusters. Four columns from the left to the right describe the event-related spectral dynamics of delta, theta, alpha, and beta bands, respectively. Top and middle panels are the spectral perturbations in the $\mathrm{K}^{-}$session and the $\mathrm{K}^{+}$session, respectively, where each colored horizontal bar represents a single trial in a time-warped spectral estimate. All of the spectral estimates are vertically stacked according to the RT from fast to slow (from bottom to top). Bottom panel 
presents the Pearson's correlation coefficient between RT and band power across every time point (FDR-adjusted $p$-value<0.001).

\section{Figure 5.}

Interaction plot for the theta power in the central component. (A) and (C) ExP interaction. (B) and (E) E $\times \mathrm{K}$ interaction. (D) and $(\mathrm{F}) \mathrm{P} \times \mathrm{K}$ interaction. Gray cell refers a significant interaction effect (Table 2). Asterisks between two levels of a factor indicate that the difference between group means by post-hoc tests is significant. Significant at FDR-adjusted $p<0.001$.

\section{Figure 6.}

Interaction plot for the alpha power in the central component. (A) and (C) ExP interaction. (B) and (E) $\mathrm{E} \times \mathrm{K}$ interaction. (D) and $(\mathrm{F}) \mathrm{P} \times \mathrm{K}$ interaction. Gray cell refers a significant interaction effect (Table 2). Asterisks between two levels of a factor indicate that the difference between group means by post-hoc tests is significant. Significant at FDR-adjusted $p<0.001$.

\section{Figure 7.}

Interaction plot for the theta power in the frontal component. (A) and (C) ExP interaction. (B) and (E) E×K interaction. (D) and (F) $\mathrm{P} \times \mathrm{K}$ interaction. Gray cell refers a significant interaction effect (Table 2). Asterisks between two levels of a factor indicate that the difference between group means by post-hoc tests is significant. Significant at FDR-adjusted $p<0.001$. 


\section{References}

Banks, S., Catcheside, P., Lack, L., Grunstein, R.R., McEvoy, R.D., 2004. Low levels of alcohol impair driving simulator performance and reduce perception of crash risk in partially sleep deprived subjects. Sleep 27, 1063-1067.

Boyle, L.N., Tippin, J., Paul, A., Rizzo, M., 2008. Driver performance in the moments surrounding a microsleep. Transp. Res. Part F Traffic Psychol. Behav. 11, 126-136.

Bremmer, F., Schlack, A., Shah, N.J., Zafiris, O., Kubischik, M., Hoffmann, K.-P., Zilles, K., Fink, G.R., 2001. Polymodal motion processing in posterior parietal and premotor cortex: a human fMRI study strongly implies equivalencies between humans and monkeys. Neuron 29, 287-296.

Calhoun, V.D., Pearlson, G.D., 2012. A selective review of simulated driving studies: Combining naturalistic and hybrid paradigms, analysis approaches, and future directions. NeuroImage $59,25-35$.

Campagne, A., Pebayle, T., Muzet, A., 2004. Correlation between driving errors and vigilance level: influence of the driver's age. Physiol. Behav. 80, 515-524.

Chee, M.W., Tan, J.C., Zheng, H., Parimal, S., Weissman, D.H., Zagorodnov, V., Dinges, D.F., 2008. Lapsing during sleep deprivation is associated with distributed changes in brain activation. J. Neurosci. 28, 5519-5528. 
Chuang, S.-W., Ko, L.-W., Lin, Y.-P., Huang, R.-S., Jung, T.-P., Lin, C.-T., 2012. Co-modulatory spectral changes in independent brain processes are correlated with task performance. NeuroImage 62, 1469-1477.

Cochin, S., Barthelemy, C., Lejeune, B., Roux, S., Martineau, J., 1998. Perception of motion and qEEG activity in human adults. Electroencephalogr. Clin. Neurophysiol. 107, 287-295.

Cruikshank, L.C., Singhal, A., Hueppelsheuser, M., Caplan, J.B., 2012. Theta oscillations reflect a putative neural mechanism for human sensorimotor integration. J. Neurophysiol. 107, 65-77.

Davidson, P.R., Jones, R.D., Peiris, M.T., 2007. EEG-based lapse detection with high temporal resolution. IEEE Trans. Biomed. Eng. 54, 832-839.

De Rosario, H., Solaz, J.S., Rodrı'guez, N., Bergasa, L.M., 2010. Controlled inducement and measurement of drowsiness in a driving simulator. IET Intell. Transp. Syst. 4, 280-288.

Delorme, A., Makeig, S., 2004. EEGLAB: An open source toolbox for analysis of single-trial EEG dynamics including independent component analysis. J. Neurosci. Methods 134, 9-21.

Deutschlander, A., Bense, S., Stephan, T., Schwaiger, M., Brandt, T., Dieterich, M., 2002. Sensory system interactions during simultaneous vestibular and visual stimulation in PET. Hum. Brain Mapp. 16, 92-103.

Elidan, J., Leibner, E., Freeman, S., Sela, M., Nitzan, M., Sohmer, H., 1991. Short and middle latency vestibular evoked responses to acceleration in man. Electroencephalogr. Clin. Neurophysiol. 80, 140-145. 
Eoh, H.J., Chung, M.K., Kim, S.-H., 2005. Electroencephalographic study of drowsiness in simulated driving with sleep deprivation. Int. J. Ind. Ergonomics 35, 307-320.

Esser, S.K., Hill, S., Tononi, G., 2009. Breakdown of effective connectivity during slow wave sleep: investigating the mechanism underlying a cortical gate using large-scale modeling. J. Neurophysiol. 102, 2096-2111.

Ferrara, M., De Gennaro, L., 2001. How much sleep do we need? Sleep Med. Rev. 5, 155-179.

Friston, K.J., Williams, S., Howard, R., Frackowiak, R.S., Turner, R., 1996. Movement-related effects in fMRI time-series. Magn. Reson. Med. 35, 346-355.

Fritz, C.O., Morris, P.E., Richler, J.J., 2012. Effect size estimates: current use, calculations, and interpretation. J Exp Psychol Gen 141, 2-18.

Gioia, A.J., Morphew, C.E., 1968. Evaluation of driver vision. General Motors Automobile Safety Seminar, pp. 1-14.

Goldman, R.I., Stern, J.M., Engel, J., Jr., Cohen, M.S., 2002. Simultaneous EEG and fMRI of the alpha rhythm. Neuroreport 13, 2487-2492.

Gomarus, H.K., Althaus, M., Wijers, A.A., Minderaa, R.B., 2006. The effects of memory load and stimulus relevance on the EEG during a visual selective memory search task: an ERP and ERD/ERS study. Clin. Neurophysiol. 117, 871-884.

Herculano-Houzel, S., Munk, M.H., Neuenschwander, S., Singer, W., 1999. Precisely synchronized oscillatory firing patterns require electroencephalographic activation. J. Neurosci. 19, 39924010. 
Holroyd, C.B., Nieuwenhuis, S., Yeung, N., Nystrom, L., Mars, R.B., Coles, M.G., Cohen, J.D., 2004. Dorsal anterior cingulate cortex shows fMRI response to internal and external error signals. Nat. Neurosci. 7, 497-498.

Huang, R.-S., Jung, T.-P., Delorme, A., Makeig, S., 2008. Tonic and phasic electroencephalographic dynamics during continuous compensatory tracking. NeuroImage 39, 1896-1909.

Huang, R.-S., Jung, T.-P., Makeig, S., 2009. Tonic changes in EEG power spectra during simulated driving. Lect. Notes Comput. Sci. 5638 LNAI, 394-403.

Jap, B.T., Lal, S., Fischer, P., 2011. Comparing combinations of EEG activity in train drivers during monotonous driving. Expert Syst. Appl. 38, 996-1003.

Jap, B.T., Lal, S., Fischer, P., Bekiaris, E., 2009. Using EEG spectral components to assess algorithms for detecting fatigue. Expert Syst. Appl. 36, 2352-2359.

Jung, T.-P., Makeig, S., McKeown, M.J., Bell, A.J., Lee, T.W., Sejnowski, T.J., 2001. Imaging brain dynamics using independent component analysis. Proc. IEEE 89, 1107-1122.

Kasschau, R.A., 1985. Psychology: Exploring Behavior, 2 ed. Pearson Prentice Hall.

Kemeny, A., Panerai, F., 2003. Evaluating perception in driving simulation experiments. Trends Cogn. Sci. 7, 31-37.

Khushaba, R.N., Kodagoda, S., Lal, S., Dissanayake, G., 2011. Driver drowsiness classification using fuzzy wavelet-packet-based feature-extraction algorithm. IEEE Trans. Biomed. Eng. $58,121-131$. 
Kim, S., Hong, J.-H., Li, K., Forlizzi, J., Dey, A., 2012. Route Guidance Modality for Elder Driver Navigation. In: Kay, J., Lukowicz, P., Tokuda, H., Olivier, P., Krüger, A. (Eds.), Pervasive Computing. Springer Berlin Heidelberg, pp. 179-196.

Klimesch, W., 1999. EEG alpha and theta oscillations reflect cognitive and memory performance: a review and analysis. Brain Res. Rev. 29, 169-195.

Klimesch, W., Sauseng, P., Hanslmayr, S., 2007. EEG alpha oscillations: the inhibition-timing hypothesis. Brain Res. Rev. 53, 63-88.

Lal, S.K.L., Craig, A., 2001. A critical review of the psychophysiology of driver fatigue. Biol. Psychol. 55, 173-194.

Lal, S.K.L., Craig, A., 2002. Driver fatigue: Electroencephalography and psychological assessment. Psychophysiology 39, 313-321.

Lal, S.K.L., Craig, A., Boord, P., Kirkup, L., Nguyen, H., 2003. Development of an algorithm for an EEG-based driver fatigue countermeasure. J. Saf. Res. 34, 321-328.

Liao, L.-D., Lin, C.-T., McDowell, K., Wickenden, A.E., Gramann, K., Jung, T.-P., Ko, L.-W., Chang, J.-Y., 2012. Biosensor technologies for augmented brain-computer interfaces in the next decades. Proc. IEEE 100, 1553-1566.

Lin, C.-T., Chuang, C.-H., Wang, Y.-K., Tsai, S.-F., Chiu, T.-C., Ko, L.-W., 2012a. Neurocognitive characteristics of the driver: A review on drowsiness, distraction, navigation, and motion sickness. Journal of Neuroscience and Neuroengineering 1, 61-81. 
Lin, C.-T., Huang, K.-C., Chao, C.-F., Chen, J.-A., Chiu, T.-W., Ko, L.-W., Jung, T.-P., 2010.

Tonic and phasic EEG and behavioral changes induced by arousing feedback. NeuroImage

$52,633-642$.

Lin, C.-T., Ko, L.-W., Chung, I.-F., Huang, T.-Y., Chen, Y.-C., Jung, T.-P., Liang, S.-F., 2006.

Adaptive EEG-based alertness estimation system by using ICA-based fuzzy neural networks.

IEEE Trans. Circuits Syst. I-Regul. Pap. 53, 2469-2476.

Lin, C.-T., Wu, R.-C., Jung, T.-P., Liang, S.-F., Huang, T.-Y., 2005. Estimating driving

performance based on EEG spectrum analysis. EURASIP J. Appl. Signal Processing 19, $3165-3174$.

Lin, F.-C., Ko, L.-W., Chuang, C.-H., Su, T.-P., Lin, C.-T., 2012b. Generalized EEG-based drowsiness prediction system by using a self-organizing neural fuzzy system. IEEE Trans.

Circuits Syst. I-Regul. Pap. 59, 2044-2055.

Loose, R., Ayan, T., Probst, T., 1999. Visual motion direction evoked potentials are direction specifically influenced by concurrent vestibular stimulation. Clin. Neurophysiol. 110, $192-$ 199.

Lopez, C., Blanke, O., 2011. The thalamocortical vestibular system in animals and humans. Brain Res. Rev. 67, 119-146.

Lotjonen, J., Korhonen, I., Hirvonen, K., Eskelinen, S., Myllymaki, M., Partinen, M., 2003. Automatic sleep-wake and nap analysis with a new wrist worn online activity monitoring device vivago WristCare. Sleep 26, 86-90. 
Luu, P., Tucker, D.M., Makeig, S., 2004. Frontal midline theta and the error-related negativity: neurophysiological mechanisms of action regulation. Clin Neurophysiol 115, 1821-1835.

Makeig, S., Inlow, M., 1993. Lapses in alertness: coherence of fluctuations in performance and EEG spectrum. Electroencephalogr Clin Neurophysiol 86, 23-35.

Makeig, S., Jung, T.P., Bell, A.J., Ghahremani, D., Sejnowski, T.J., 1997. Blind separation of auditory event-related brain responses into independent components. Proc. Natl. Acad. Sci. U.S.A. 94, 10979-10984.

Makeig, S., Westerfield, M., Jung, T.P., Enghoff, S., Townsend, J., Courchesne, E., Sejnowski, T.J., 2002. Dynamic brain sources of visual evoked responses. Science 295, 690-694.

Marco-Pallares, J., Grau, C., Ruffini, G., 2005. Combined ICA-LORETA analysis of mismatch negativity. NeuroImage 25, 471-477.

Massimini, M., Ferrarelli, F., Huber, R., Esser, S.K., Singh, H., Tononi, G., 2005. Breakdown of cortical effective connectivity during sleep. Science 309, 2228-2232.

Milne, E., Scope, A., Pascalis, O., Buckley, D., Makeig, S., 2009. Independent component analysis reveals atypical electroencephalographic activity during visual perception in individuals with autism. Biol Psychiatry 65, 22-30.

Nehmeh, S.A., Erdi, Y.E., 2008. Respiratory motion in positron emission tomography/computed tomography: a review. Semin. Nucl. Med. 38, 167-176.

Neubauer, C., Matthews, G., Langheim, L., Saxby, D., 2012. Fatigue and voluntary utilization of automation in simulated driving. Hum. Factors 54, 734-746. 
Ngo, M.K., Pierce, R.S., Spence, C., 2012. Using multisensory cues to facilitate air traffic management. Hum. Factors 54, 1093-1103.

Nguyen, T., Hau, R., Bartlett, J., 2000. Driving reaction time before and after anterior cruciate ligament reconstruction. Knee Surg. Sports Traumatol. Arthrosc. 8, 226-230.

Nolan, H., Butler, J.S., Whelan, R., Foxe, J.J., Bulthoff, H.H., Reilly, R.B., 2012. Neural correlates of oddball detection in self-motion heading: a high-density event-related potential study of vestibular integration. Exp. Brain Res. 219, 1-11.

Onton, J., Delorme, A., Makeig, S., 2005. Frontal midline EEG dynamics during working memory. NeuroImage 27, 341-356.

Oostenveld, R., Oostendorp, T.F., 2002. Validating the boundary element method for forward and inverse EEG computations in the presence of a hole in the skull. Hum. Brain Mapp. 17, 179192.

Otmani, S., Pebayle, T., Roge, J., Muzet, A., 2005. Effect of driving duration and partial sleep deprivation on subsequent alertness and performance of car drivers. Physiol. Behav. 84, 715-724.

Peiris, M.T., Jones, R.D., Davidson, P.R., Carroll, G.J., Bones, P.J., 2006. Frequent lapses of responsiveness during an extended visuomotor tracking task in non-sleep-deprived subjects. J. Sleep Res. 15, 291-300. 
Perfetti, B., Moisello, C., Landsness, E.C., Kvint, S., Pruski, A., Onofrj, M., Tononi, G., Ghilardi, M.F., 2011. Temporal evolution of oscillatory activity predicts performance in a choicereaction time reaching task. J. Neurophysiol. 105, 18-27.

Probst, T., Ayan, T., Loose, R., Skrandies, W., 1997. Electrophysiological evidence for directionspecific rotary evoked potentials in human subjects-a topographical study. Neurosci. Lett. 239, 97-100.

Rawle, C.J., Miall, R.C., Praamstra, P., 2012. Frontoparietal theta activity supports behavioral decisions in movement-target selection. Front. Hum. Neurosci. 6, 1-11.

Rodionov, V., Elidan, J., Sela, M., Nitzan, M., Sohmer, H., 1996. Vertical plane short and middle latency vestibular evoked potentials in humans. Ann. Otol. Rhinol. Laryngol. 105, 43-48.

Sarter, M., Givens, B., Bruno, J.P., 2001. The cognitive neuroscience of sustained attention: where top-down meets bottom-up. Brain Res. Rev. 35, 146-160.

Sauseng, P., Hoppe, J., Klimesch, W., Gerloff, C., Hummel, F.C., 2007. Dissociation of sustained attention from central executive functions: local activity and interregional connectivity in the theta range. Eur. J. Neurosci. 25, 587-593.

Sauseng, P., Klimesch, W., Schabus, M., Doppelmayr, M., 2005. Fronto-parietal EEG coherence in theta and upper alpha reflect central executive functions of working memory. Int. J. Psychophysiol. 57, 97-103. 
Scheef, L., Boecker, H., Daamen, M., Fehse, U., Landsberg, M.W., Granath, D.-O., Mechling, H., Effenberg, A.O., 2009. Multimodal motion processing in area V5/MT: Evidence from an artificial class of audio-visual events. Brain Res. 1252, 94-104.

Sivak, M., 1996. The information that drivers use: Is it indeed 90\% visual? Perception 4, 169-193.

Triggs, T.J., Harris, W.G., 1982. Reaction time of drivers to road stimuli. Monash University, Melbourne, Australia.

van Veen, V., Carter, C.S., 2002. The anterior cingulate as a conflict monitor: fMRI and ERP studies. Physiol. Behav. 77, 477-482.

Wallis, G., Chatziastros, A., Tresilian, J., Tomasevic, N., 2007. The role of visual and nonvisual feedback in a vehicle steering task. J. Exp. Psychol. Hum. Percept. Perform. 33, 1127-1144.

Young, M.S., Stanton, N.A., 2007. Back to the future: brake reaction times for manual and automated vehicles. Ergonomics 50, 46-58.

Zhang, Y., Wang, X., Bressler, S.L., Chen, Y., Ding, M., 2008. Prestimulus cortical activity is correlated with speed of visuomotor processing. J. Cogn. Neurosci. 20, 1915-1925. 


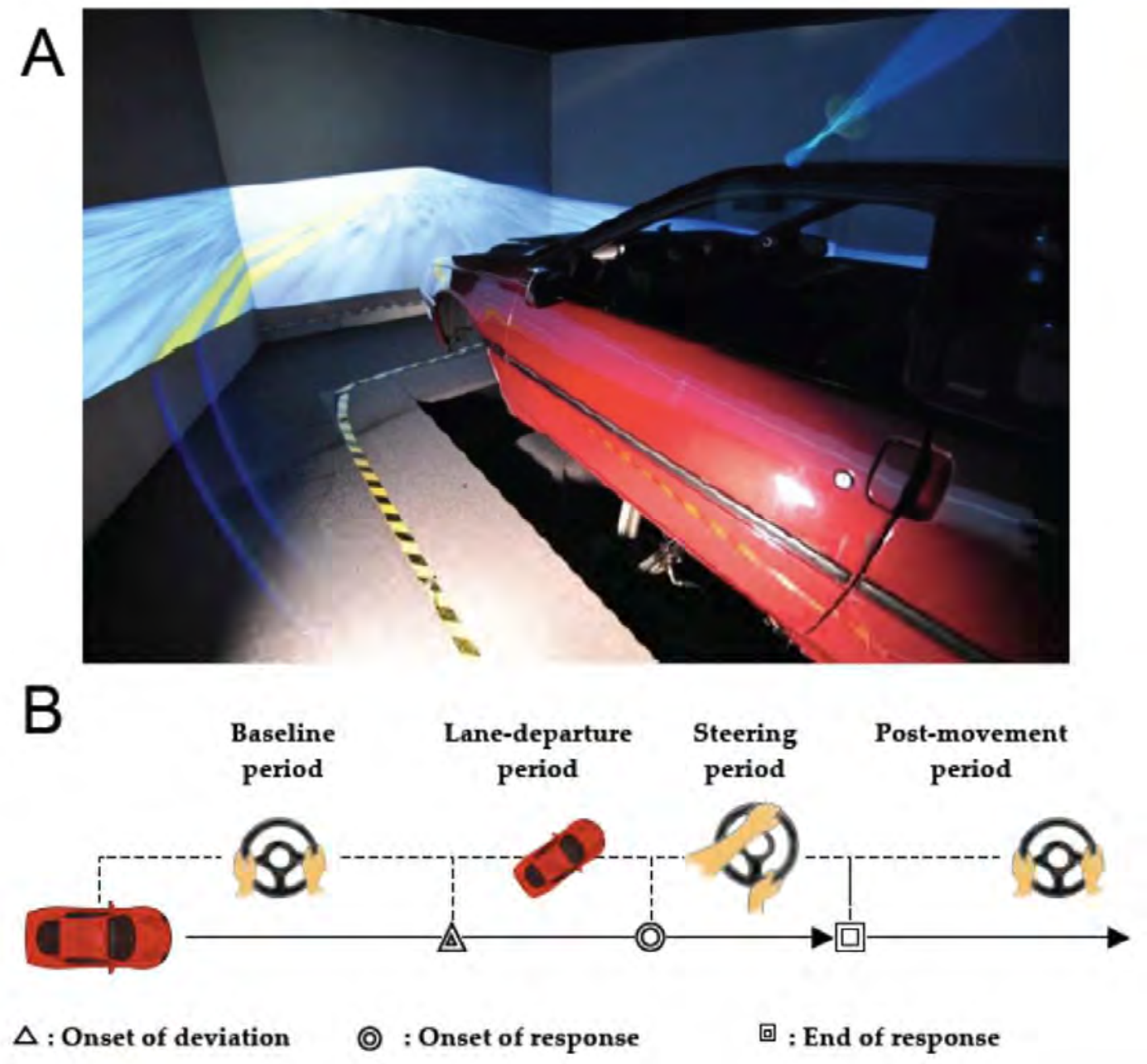

Fig. 1 

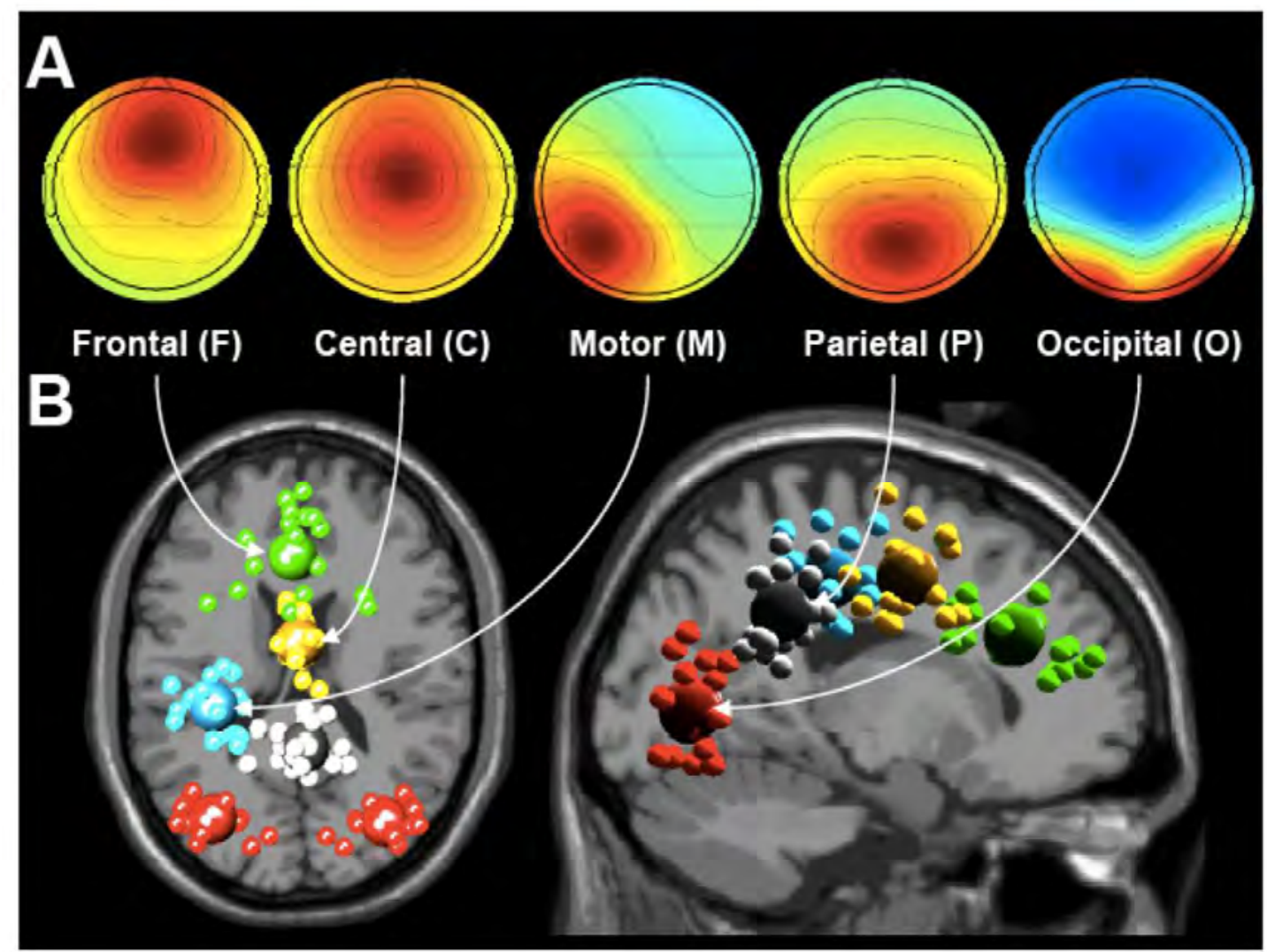

Fig. 2 
A

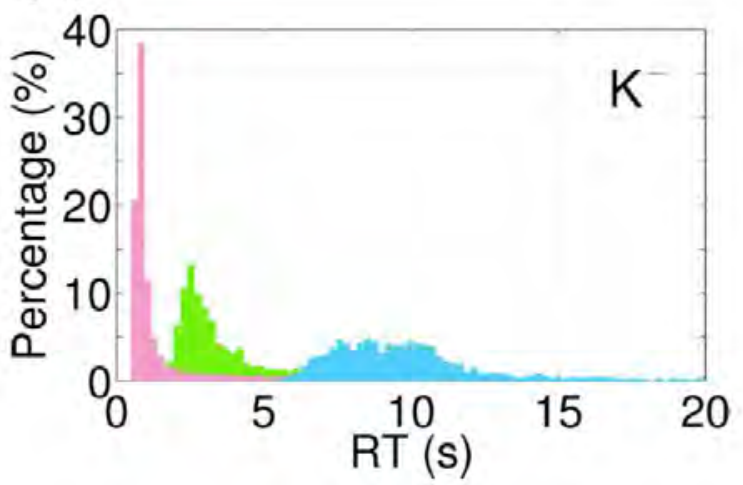

B

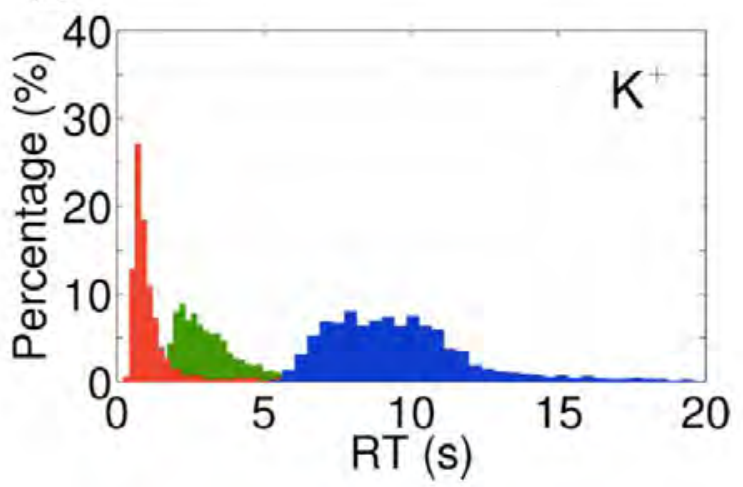

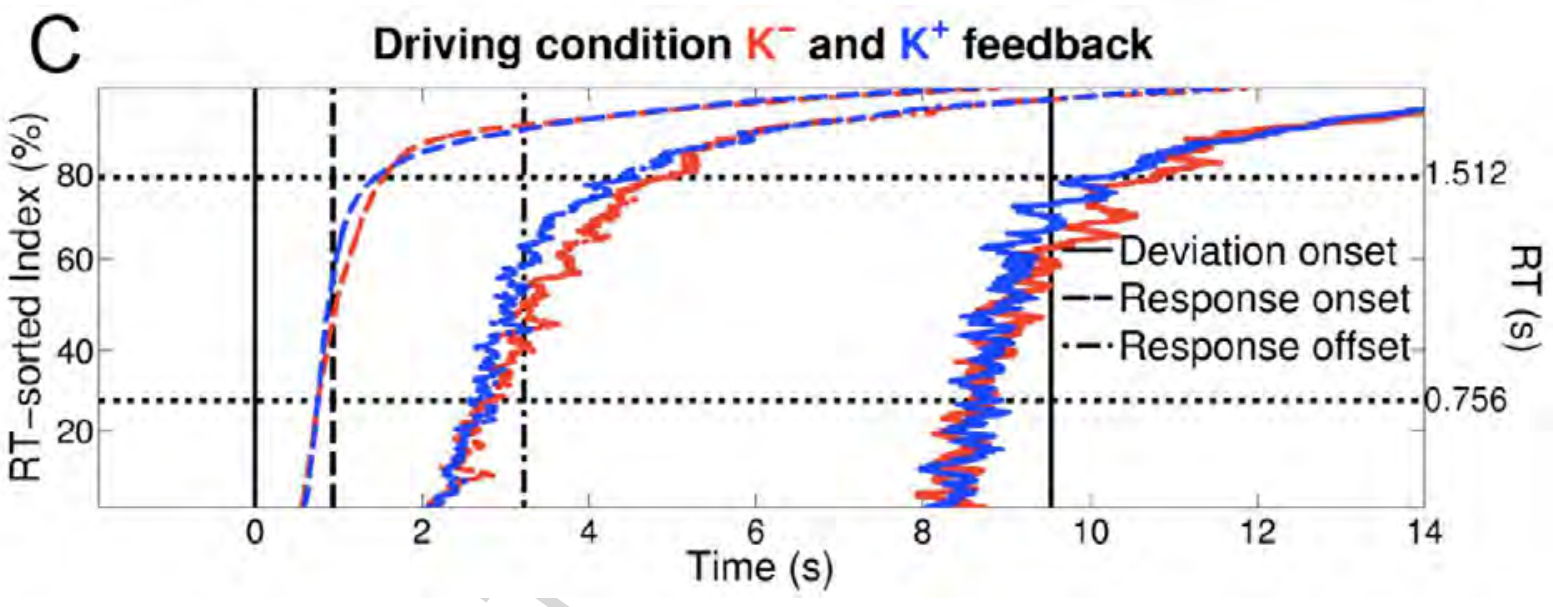

Fig. 3 


\section{A}

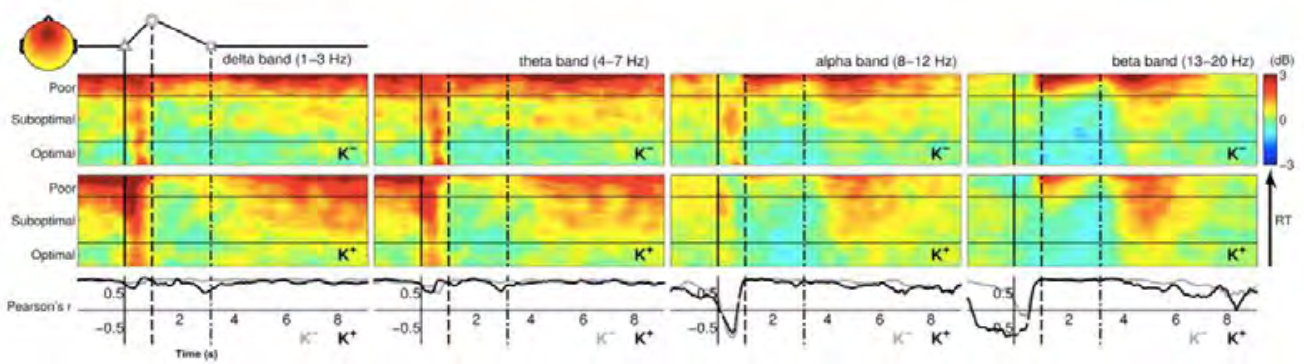

B

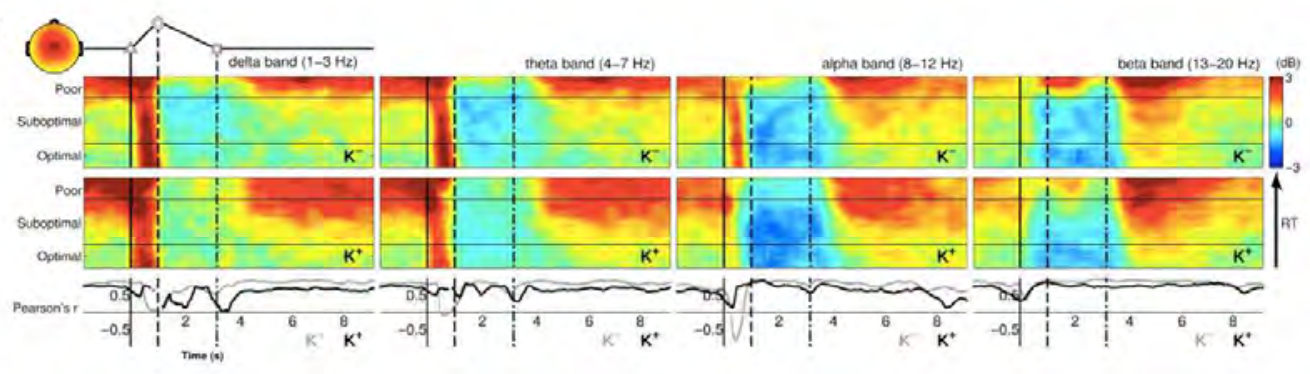

C

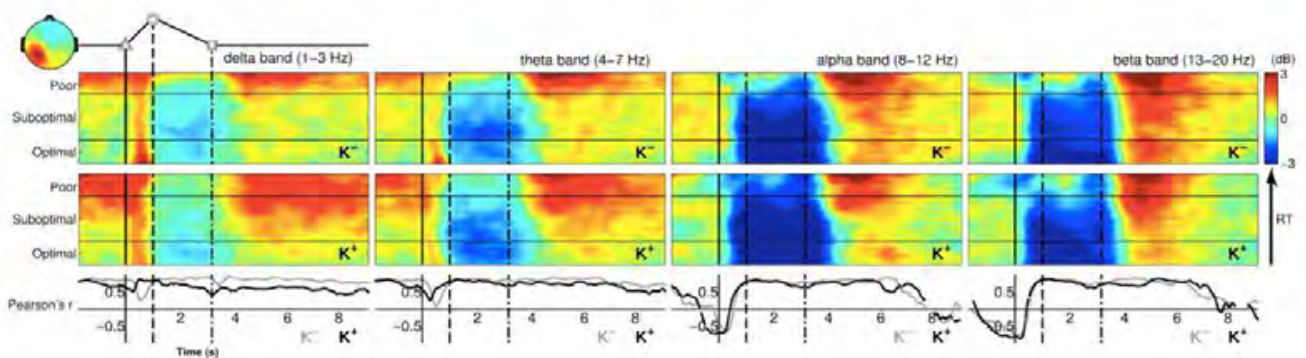

D

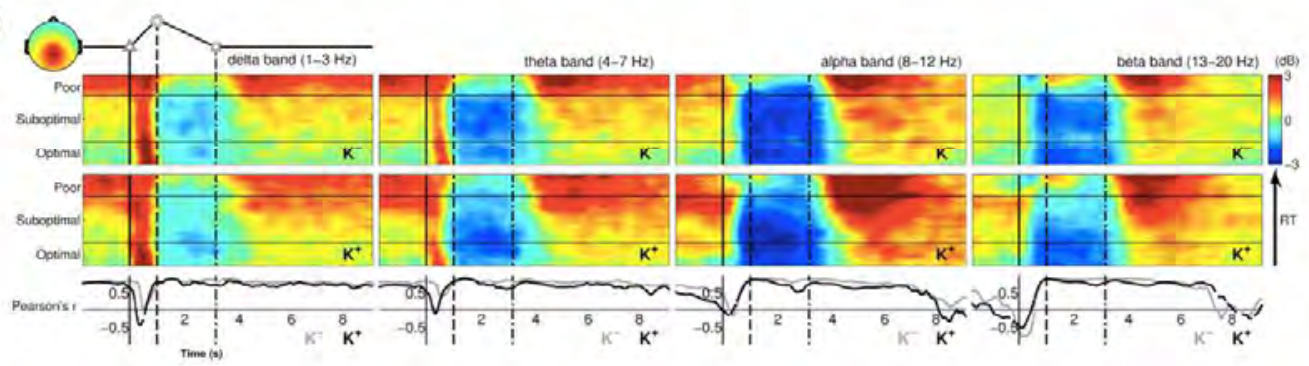

E

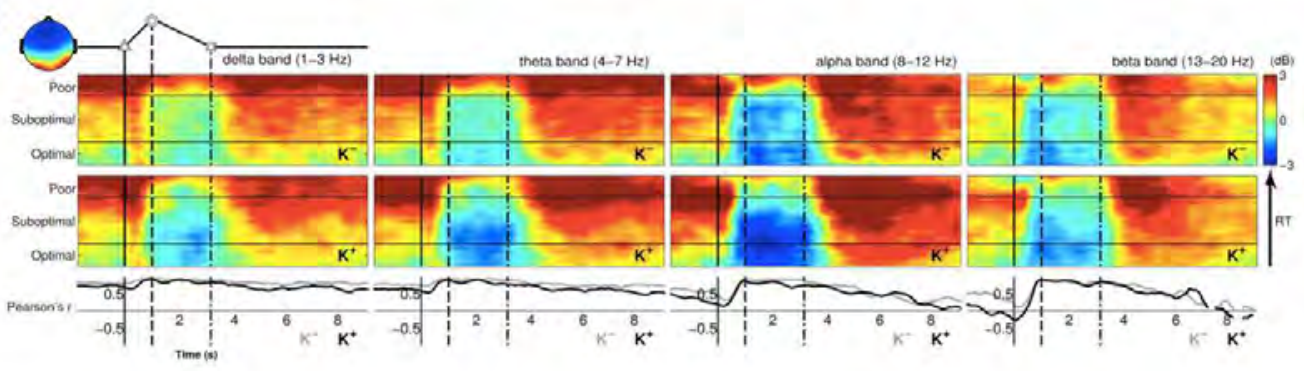

Fig. 4 


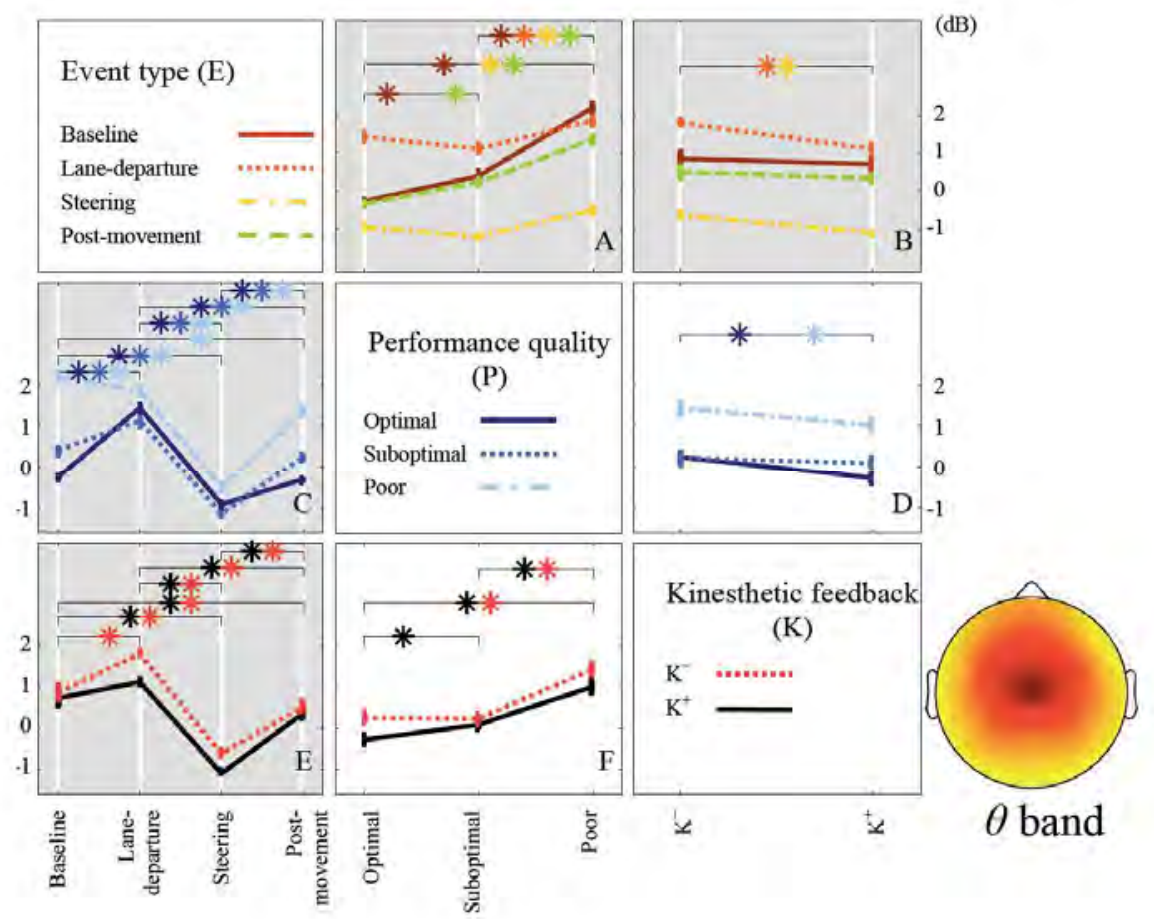

Fig. 5 


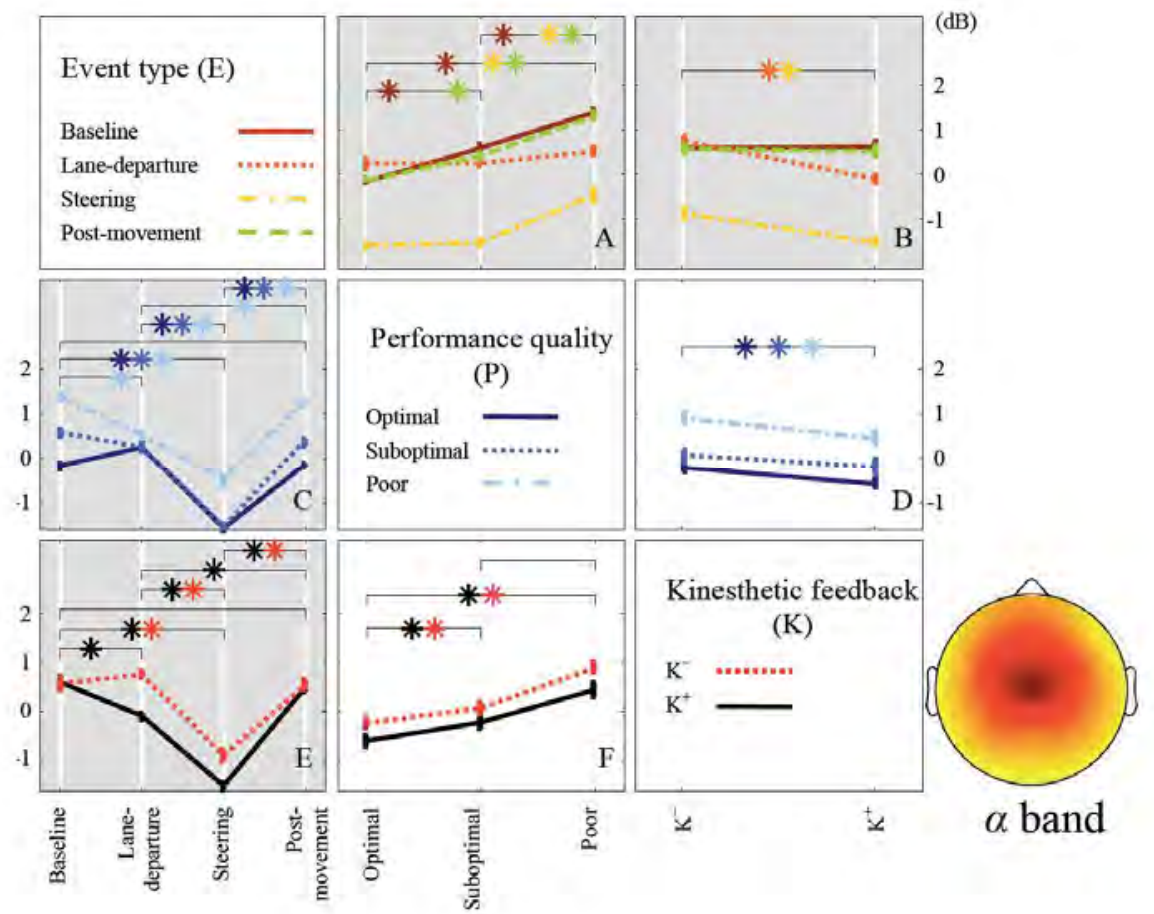

Fig. 6 


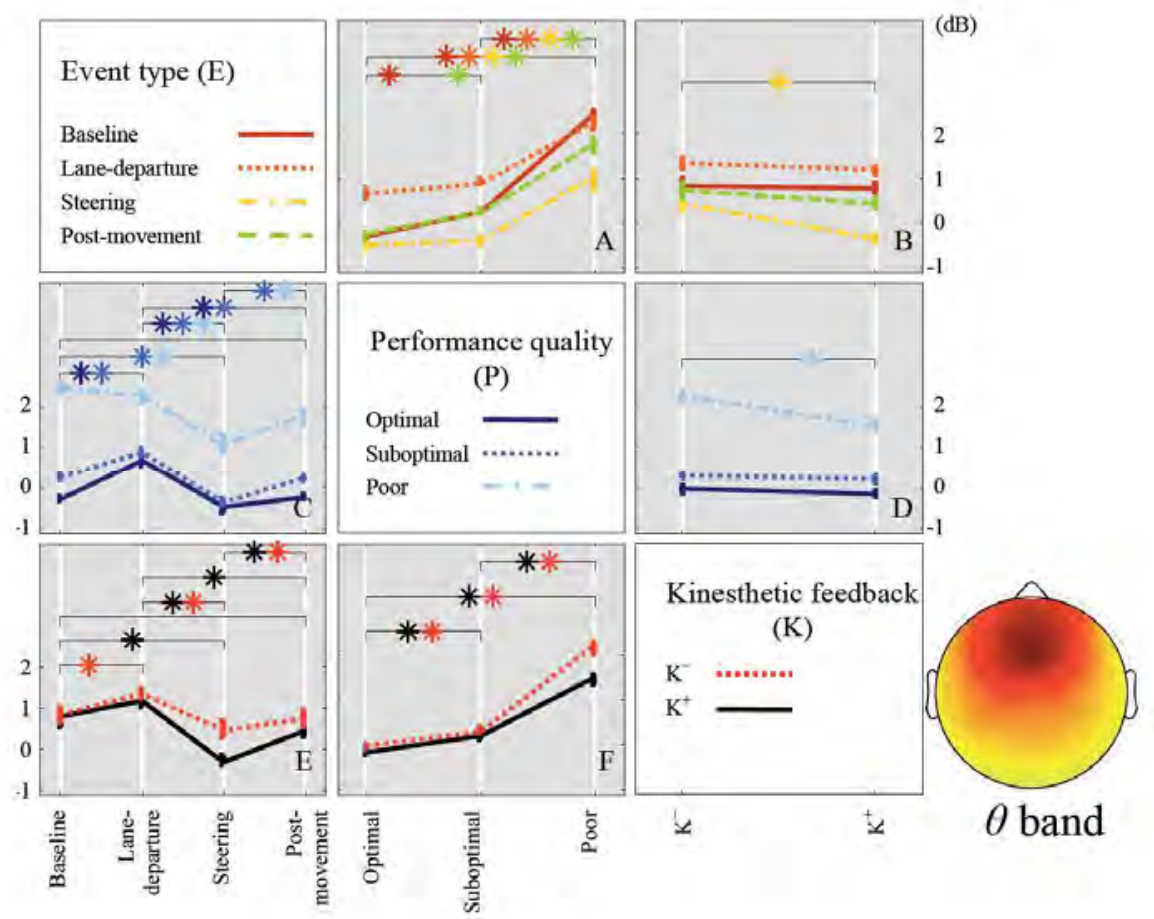

Fig. 7 
Table 1. Summary of trials collected from each subject

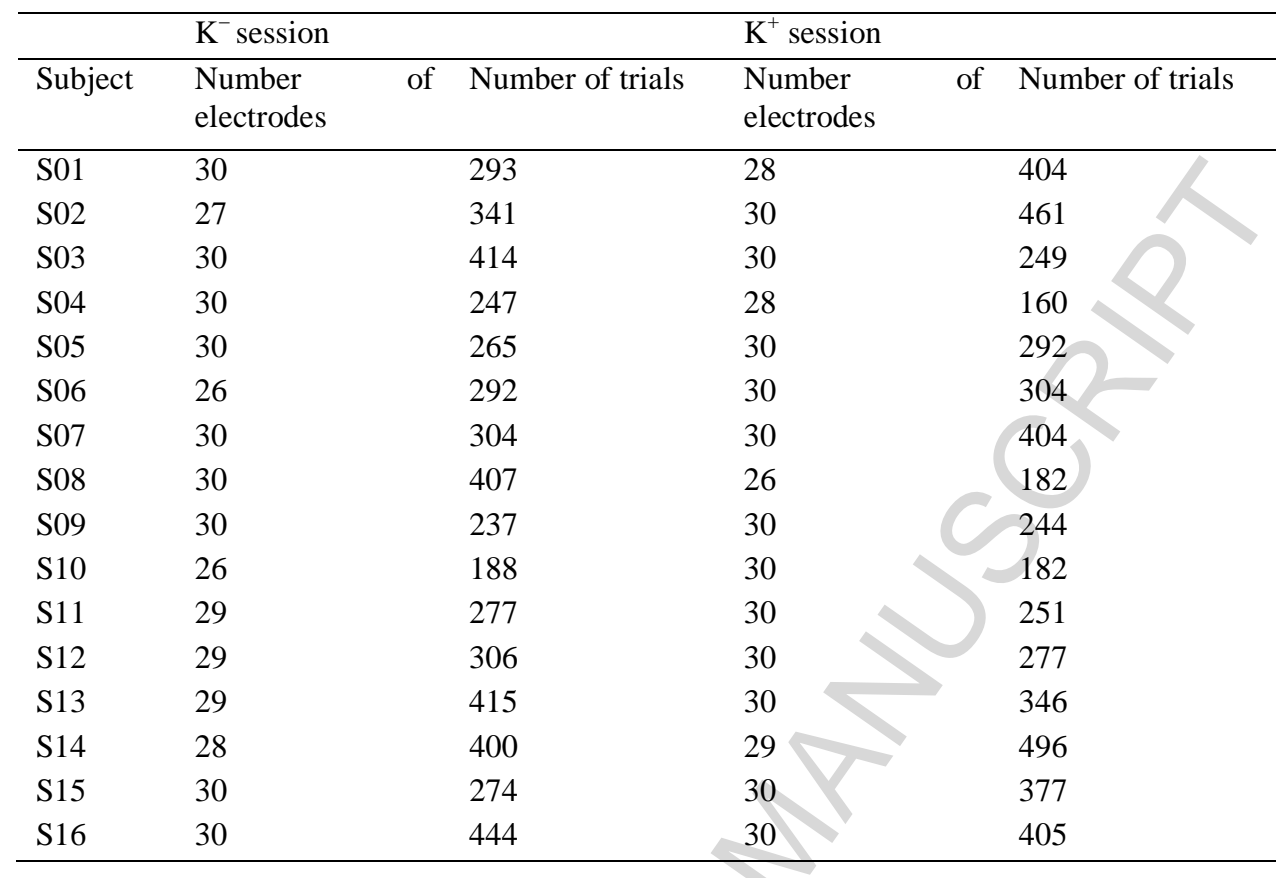


Table 2. Results of three-way repeated measure ANOVA.

\begin{tabular}{|c|c|c|c|c|c|c|c|c|}
\hline \multirow{2}{*}{$\begin{array}{l}\text { Independent } \\
\text { component }\end{array}$} & \multirow{2}{*}{$\begin{array}{l}\text { Spectral } \\
\text { band }\end{array}$} & \multicolumn{3}{|l|}{ Main effect } & \multicolumn{4}{|c|}{ Interaction effect } \\
\hline & & $\begin{array}{l}\text { Event type } \\
\text { (E) }\end{array}$ & $\begin{array}{l}\text { Performance } \\
\text { quality }(\mathrm{P})\end{array}$ & $\begin{array}{l}\text { Kinesthestic } \\
\text { feedback }(K)\end{array}$ & $\mathrm{E} \times \mathrm{P}$ & $\mathrm{E} \times \mathrm{K}$ & $\mathrm{P} \times \mathrm{K}$ & $\mathrm{E} \times \mathrm{P} \times \mathrm{K}$ \\
\hline \multirow{8}{*}{ Frontal } & \multirow{2}{*}{$\delta$} & $p<0.001$ & $p<0.001$ & $p<0.001$ & $p<0.001$ & $p<0.001$ & n.s. & $p<0.001$ \\
\hline & & $\eta_{p}^{2}=0.89$ & $\eta_{p}^{2}=0.84$ & $\eta_{p}^{2}=0.82$ & $\eta_{p}^{2}=0.54$ & $\eta_{p}^{2}=0.54$ & 8 & $\eta_{p}^{2}=0.32$ \\
\hline & \multirow{2}{*}{$\theta$} & $p<0.001$ & $p<0.001$ & $p<0.001$ & $p<0.001$ & $p<0.001$ & $p<0.001$ & n.s. \\
\hline & & $\eta_{p}^{2}=0.83$ & $\eta_{p}^{2}=0.85$ & $\eta_{p}^{2}=0.87$ & $\eta_{p}^{2}=0.54$ & $\eta_{p}^{2}=0.43$ & $\eta_{p}^{2}=0.48$ & \\
\hline & \multirow{2}{*}{$\alpha$} & $p<0.001$ & $p<0.001$ & $p<0.001$ & $p<0.001$ & $p<0.001$ & $p<0.001$ & n.s. \\
\hline & & $\eta_{p}^{2}=0.40$ & $\eta_{p}^{2}=0.83$ & $\eta_{p}^{2}=0.76$ & $\eta_{p}^{2}=0.67$ & $\eta_{p}^{2}=0.42$ & $\eta_{p}^{2}=0.48$ & \\
\hline & \multirow{2}{*}{$\beta$} & $p<0.001$ & $p<0.001$ & n.s. & $p<0.001$ & $p<0.001$ & $p<0.001$ & n.s. \\
\hline & & $\eta_{p}^{2}=0.49$ & $\eta_{p}^{2}=0.82$ & & $\eta_{p}^{2}=0.76$ & $\eta_{p}^{2}=0.35$ & $\eta_{p}^{2}=0.52$ & \\
\hline \multirow{8}{*}{ Central } & \multirow{2}{*}{$\delta$} & $p<0.001$ & $p<0.001$ & $p<0.001$ & $p<0.001$ & n.s. & n.s. & n.s. \\
\hline & & $\eta_{p}^{2}=0.97$ & $\eta_{p}^{2}=0.90$ & $\eta_{p}^{2}=0.86$ & $\eta_{p}^{2}=0.8416$ & & & \\
\hline & \multirow{2}{*}{$\theta$} & $p=0.000$ & $p<0.001$ & $p<0.001$ & $p<0.001$ & $p<0.001$ & n.s. & n.s. \\
\hline & & $\eta_{p}^{2}=0.98$ & $\eta_{p}^{2}=0.91$ & $\eta_{p}^{2}=0.92$ & $\eta_{p}^{2}=0.87$ & $\eta_{p}^{2}=0.62$ & & \\
\hline & \multirow{2}{*}{$\alpha$} & $p<0.001$ & $p<0.001$ & $p<0.001$ & $p<0.001$ & $p<0.001$ & n.s. & $p<0.001$ \\
\hline & & $\eta_{p}^{2}=0.99$ & $\eta_{p}^{2}=0.90$ & $\eta_{p}^{2}=0.85$ & $\eta_{p}^{2}=0.71$ & $\eta_{p}^{2}=0.86$ & & $\eta_{p}^{2}=0.39$ \\
\hline & \multirow{2}{*}{$\beta$} & $p<0.001$ & $p<0.001$ & n.s. & $p<0.001$ & $p<0.001$ & n.s. & n.s. \\
\hline & & $\eta_{p}^{2}=0.96$ & $\eta_{p}^{2}=0.94$ & + & $\eta_{p}^{2}=0.76$ & $\eta_{p}^{2}=0.53$ & & \\
\hline \multirow{7}{*}{ Motor } & \multirow{2}{*}{$\delta$} & $p<0.001$ & $p<0.001$ & $p<0.001$ & $p<0.001$ & $p<0.001$ & n.s. & $p<0.001$ \\
\hline & & $\eta_{p}^{2}=0.97$ & $\eta_{p}^{2}=0.89$ & $\eta_{p}^{2}=0.81$ & $\eta_{p}^{2}=0.66$ & $\eta_{p}^{2}=0.48$ & & $\eta_{p}^{2}=0.23$ \\
\hline & \multirow{2}{*}{$\theta$} & $P<0.001$ & $p<0.001$ & n.s. & $p<0.001$ & $p<0.001$ & n.s. & n.s. \\
\hline & & $\eta_{p}^{2}=0.97$ & $\eta_{p}^{2}=0.88$ & & $\eta_{p}^{2}=0.65$ & $\eta_{p}^{2}=0.56$ & & \\
\hline & \multirow{2}{*}{$\alpha$} & $p<0.001$ & $p<0.001$ & $p<0.001$ & $p<0.001$ & $p<0.001$ & n.s. & n.s. \\
\hline & & $r_{p}^{2}=0.98$ & $r_{p}^{2}=0.84$ & $\eta_{p}^{2}=0.67$ & $r_{p}^{2}=0.72$ & $r_{p}^{2}=0.50$ & & \\
\hline & $\beta$ & $\begin{array}{l}p<0.001 \\
\eta_{p}^{2}=0.98\end{array}$ & $\begin{array}{l}P<0.001 \\
\eta_{p}^{2}=0.86\end{array}$ & n.s. & $\begin{array}{l}p<0.001 \\
\eta_{p}^{2}=0.86\end{array}$ & $\begin{array}{l}p<0.001 \\
\eta_{p}^{2}=0.45\end{array}$ & n.s. & n.s. \\
\hline \multirow{8}{*}{ Parietal } & \multirow{2}{*}{$\delta$} & $p<0.001$ & $p<0.001$ & $p<0.001$ & $p<0.001$ & $p<0.001$ & $p<0.001$ & n.s. \\
\hline & & $\eta_{p}^{2}=0.98$ & $\eta_{p}^{2}=0.91$ & $\eta^{2}=0.72$ & $\eta_{p}^{2}=0.85$ & $r_{p}^{2}=0.35$ & $\eta_{p}^{2}=0.40$ & \\
\hline & \multirow{2}{*}{$\theta$} & $p<0.001$ & $p<0.001$ & n.s. & $p<0.001$ & $p<0.001$ & n.s. & n.s. \\
\hline & & $\eta_{p}^{2}=0.98$ & $\eta_{p}^{2}=0.93$ & & $\eta_{p}^{2}=0.76$ & $\eta_{p}^{2}=0.42$ & & \\
\hline & \multirow{2}{*}{$\alpha$} & $P<0.001$ & $p<0.001$ & n.s. & $p<0.001$ & $p<0.001$ & n.s. & n.s. \\
\hline & & $\eta_{p}^{2}=0.98$ & $\eta_{p}^{2}=0.90$ & & $\eta_{p}^{2}=0.37$ & $\eta_{p}^{2}=0.53$ & & \\
\hline & \multirow{2}{*}{$\beta$} & $P<0.001$ & $p<0.001$ & n.s. & $p<0.001$ & n.s. & n.s. & n.s. \\
\hline & & $\eta_{p}^{2}=0.96$ & $\eta_{p}^{2}=0.95$ & & $\eta_{p}^{2}=0.22$ & & & \\
\hline & $\delta$ & $p<0.001$ & $p<0.001$ & $p<0.001$ & $p<0.001$ & $p<0.001$ & $p<0.001$ & n.s. \\
\hline & & $\eta_{p}^{2}=0.98$ & $\eta_{p}^{2}=0.97$ & $\eta_{p}^{2}=0.77$ & $\eta_{p}^{2}=0.48$ & $\eta_{p}^{2}=0.59$ & $\eta_{p}^{2}=0.40$ & \\
\hline & $\theta$ & $p<0.001$ & $p<0.001$ & $p<0.001$ & $p<0.001$ & $P<0.001$ & n.s. & n.s. \\
\hline & $\nabla$ & $\eta_{p}^{2}=0.98$ & $\eta_{p}^{2}=0.98$ & $\eta_{p}^{2}=0.61$ & $\eta_{p}^{2}=0.39$ & $\eta_{p}^{2}=0.63$ & & \\
\hline Occipital & $\alpha$ & $p<0.001$ & $p<0.001$ & n.s. & n.s. & $p<0.001$ & n.s. & n.s. \\
\hline & $\alpha$ & $\eta_{p}^{2}=0.97$ & $\eta_{p}^{2}=0.91$ & & & $\eta_{p}^{2}=0.69$ & & \\
\hline & $\beta$ & $p<0.001$ & $p<0.001$ & n.s. & $p<0.001$ & $p<0.001$ & n.s. & $p<0.001$ \\
\hline & $\rho$ & $\eta_{p}^{2}=0.96$ & $\eta_{p}^{2}=0.88$ & & $\eta_{p}^{2}=0.43$ & $\eta_{p}^{2}=0.45$ & & $\eta_{p}^{2}=0.32$ \\
\hline
\end{tabular}


Table 3. Comparison of marginal means between event types by post-hoc t-tests

\begin{tabular}{|c|c|c|c|c|c|c|c|c|c|c|c|c|}
\hline & \multicolumn{4}{|c|}{ Lane-departure vs. baseline } & \multicolumn{4}{|c|}{ Steering vs. baseline } & \multicolumn{4}{|c|}{ Post-movement vs. baseline } \\
\hline & $\delta$ & $\theta$ & $\alpha$ & $\beta$ & $\delta$ & $\theta$ & $\alpha$ & $\beta$ & $\delta$ & $\theta$ & $\alpha$ & $\beta$ \\
\hline Frontal & + & & & & - & 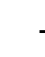 & & & & & & + \\
\hline Central & + & + & - & - & - & . & & & & & & + \\
\hline Motor & + & & - & - & - & 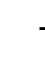 & & & & & & + \\
\hline Parietal & + & & - & - & - & 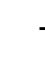 & & & & & & + \\
\hline Occipital & & - & - & - & - & 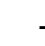 & & & & & + & + \\
\hline
\end{tabular}

Symbols + / - indicate that EEG synchrony/desynchronization is significantly greater during the lane-departure period (the steering period/the post-movement period) than that during the baseline period (FDR-adjusted $p$ value<0.001). 
Table 4. Comparison of marginal means between performance qualities by post-hoc t-tests

\begin{tabular}{|c|c|c|c|c|c|c|c|c|c|c|c|c|}
\hline & \multicolumn{4}{|c|}{ Optimal < suboptimal } & \multicolumn{4}{|c|}{ Suboptimal < poor } & \multicolumn{4}{|c|}{ Optimal < poor } \\
\hline & $\delta$ & $\theta$ & $\alpha$ & $\beta$ & $\delta$ & $\theta$ & $\alpha$ & $\beta$ & $\delta$ & $\theta$ & $\alpha$ & $\beta$ \\
\hline Frontal & & & * & & * & * & * & * & & * & * & * \\
\hline Central & & & & * & * & * & * & * & & * & * & * \\
\hline Motor & & & & * & * & * & * & * & * & * & * & * \\
\hline Parietal & & & * & * & * & * & * & & & * & * & * \\
\hline Occipital & * & * & * & * & * & * & * & & * & * & * & * \\
\hline
\end{tabular}

Symbol * refers to a situation in which the difference of power spectra between groups (i.e. optimal<suboptimal, suboptimal<poor, and optimal<poor) is statistically significant (FDR-adjusted $p$ value<0.001). 
Table 5. Comparison of marginal means between kinesthetic feedbacks by post-hoc t-tests

\begin{tabular}{lllll}
\hline & \multicolumn{4}{l}{$\mathrm{K}^{+}$vs. $\mathrm{K}^{-}$} \\
\cline { 2 - 5 } & $\delta$ & $\theta$ & $\alpha$ & $\beta$ \\
\hline Frontal & - & - & - & \\
Central & - & - & - & \\
Motor & - & & - & \\
Parietal & - & & & \\
Occipital & - & - & & \\
\hline
\end{tabular}

Symbol - indicates that the spectral power of group $\mathrm{K}^{+}$ is significantly less than that of group $\mathrm{K}^{-}$. 
Table 6. Significant event-related spectral perturbation across different levels of performance suggested by post-hoc comparisons

\begin{tabular}{|c|c|c|c|c|c|c|c|c|c|c|c|c|c|}
\hline & & \multicolumn{4}{|c|}{$\begin{array}{l}\text { Lane-departure } \\
\text { vs. baseline }\end{array}$} & \multicolumn{4}{|c|}{$\begin{array}{l}\text { Steering } \\
\text { vs. baseline }\end{array}$} & \multicolumn{4}{|c|}{$\begin{array}{l}\text { Post-movement } \\
\text { vs. baseline }\end{array}$} \\
\hline & & $\delta$ & $\theta$ & $\alpha$ & $\beta$ & $\delta$ & $\theta$ & & $\beta$ & $\delta$ & $\theta$ & $\alpha$ & $\beta$ \\
\hline \multirow[t]{5}{*}{ Optimal } & Frontal & + & + & & & & & & & & & & \\
\hline & Central & + & + & & - & - & & & & & & & \\
\hline & Motor & + & + & - & - & - & & & & & & & \\
\hline & Parietal & + & + & - & - & - & & & & & & & + \\
\hline & Occipital & + & & - & - & - & 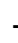 & & - & + & + & + & + \\
\hline \multirow[t]{5}{*}{ Suboptimal } & Frontal & + & + & & & - & & & - & & & & + \\
\hline & Central & + & + & & - & & & & - & & & & + \\
\hline & Motor & + & - & - & - & & & & - & & & & \\
\hline & Parietal & + & & - & . & & & & - & & & & \\
\hline & Occipital & & - & - & - & - & . & & - & & & & + \\
\hline \multirow[t]{5}{*}{ Poor } & Frontal & & & - & & - & - & & + & - & & + & + \\
\hline & Central & & & - & & - & . & & & - & - & & + \\
\hline & Motor & & - & & 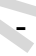 & - & . & & & - & & + & + \\
\hline & Parietal & & & & - & - & . & & & - & - & + & + \\
\hline & Occipital & & & & - & - & . & & & - & - & & + \\
\hline
\end{tabular}

Symbols $+/$ - indicate that EEG synchrony/desynchronization is significantly greater during the lane-departure period (the steering period/the post-movement period) than that during the baseline period (FDR-adjusted $p$-value $<0.001$ ). 
Table 7. Significant performance-related spectral perturbation across different type of event suggested by post-hoc comparisons

\begin{tabular}{|c|c|c|c|c|c|c|c|c|c|c|c|c|c|}
\hline & & \multicolumn{4}{|c|}{ Optimal < suboptimal } & \multicolumn{4}{|c|}{ Suboptimal < poor } & \multicolumn{4}{|c|}{ Optimal < poor } \\
\hline & & $\delta$ & $\theta$ & $\alpha$ & $\beta$ & $\delta$ & $\theta$ & $\alpha$ & $\beta$ & $\delta$ & $\theta$ & $\alpha$ & $\beta$ \\
\hline \multirow{5}{*}{ 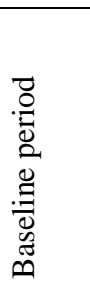 } & Frontal & * & * & * & & * & * & * & & * & * & * & \\
\hline & Central & * & * & * & * & * & * & * & * & * & * & * & * \\
\hline & Motor & * & * & & * & * & * & & & & * & & \\
\hline & Parietal & * & * & * & * & * & * & & & * & * & * & \\
\hline & Occipital & * & * & * & * & * & * & & & * & * & * & * \\
\hline \multirow{5}{*}{ 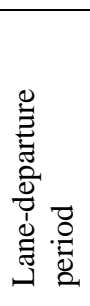 } & Frontal & & & & & * & & & * & * & * & & * \\
\hline & Central & & & & * & * & & & * & * & & & * \\
\hline & Motor & & & & * & * & & & & & & * & * \\
\hline & Parietal & & & & * & * & * & & & & * & * & * \\
\hline & Occipital & * & * & * & * & & * & & & * & * & * & * \\
\hline \multirow{5}{*}{ 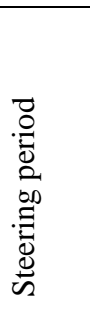 } & Frontal & & & & & & * & * & * & * & * & * & * \\
\hline & Central & & & & & & * & * & * & * & * & * & * \\
\hline & Motor & & & & & * & * & * & * & * & * & * & * \\
\hline & Parietal & & & & & * & * & * & * & * & * & * & * \\
\hline & Occipital & * & & & & * & * & * & * & * & * & * & * \\
\hline \multirow{5}{*}{ 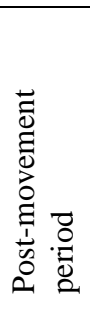 } & Frontal & * & & * & & * & * & * & * & * & * & * & * \\
\hline & Central & * & & * & * & * & * & * & * & * & * & * & * \\
\hline & Motor & 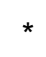 & & & * & * & * & * & * & * & * & * & * \\
\hline & Parietal & * & & * & * & * & * & * & * & * & * & * & * \\
\hline & Occipital & * & * & * & * & * & * & * & * & * & * & * & * \\
\hline
\end{tabular}

Symbol * refers to a situation in which the difference of power spectra between groups (i.e., optimal<suboptimal, suboptimal<poor, and optimal<poor) is statistically significant (FDR-adjusted $p$ value<0.001). 
Table 8. Significant event-related spectral perturbation in $\mathrm{K}^{-}$and $\mathrm{K}^{+}$sessions suggested by post-hoc comparisons

\begin{tabular}{|c|c|c|c|c|c|c|c|c|c|c|c|c|c|}
\hline & & \multicolumn{4}{|c|}{$\begin{array}{l}\text { Lane-departure period } \\
\text { vs. baseline period }\end{array}$} & \multicolumn{4}{|c|}{$\begin{array}{l}\text { Steering period } \\
\text { vs. baseline period }\end{array}$} & \multicolumn{4}{|c|}{$\begin{array}{l}\text { Post-movement period } \\
\text { vs. baseline period }\end{array}$} \\
\hline & & $\delta$ & $\theta$ & $\alpha$ & $\beta$ & $\delta$ & $\theta$ & $\alpha$ & $\beta$ & $\delta$ & $\theta$ & $\alpha$ & $\beta$ \\
\hline \multirow[t]{5}{*}{$\mathrm{K}^{-}$} & Frontal & + & + & & & - & & & & & & & + \\
\hline & Central & + & + & & - & - & - & - & & & - & & + \\
\hline & Motor & + & & - & - & - & - & - & & & & & + \\
\hline & Parietal & + & & - & - & - & - & & & & & & + \\
\hline & Occipital & & & - & - & - & - & & & & & & + \\
\hline \multirow[t]{5}{*}{$\mathrm{K}^{+}$} & Frontal & & & & - & - & & - & & & & & + \\
\hline & Central & + & & - & - & - & & & - & - & - & & + \\
\hline & Motor & & - & - & - & - & & & - & & & & + \\
\hline & Parietal & + & & - & - & & & - & - & & & & + \\
\hline & Occipital & & - & - & - & - & - & - & - & & & + & + \\
\hline
\end{tabular}

Symbols $+/$ - indicate that EEG synchrony/desynchronization is significantly greater during the lane-departure period (the steering period/the post-movement period) than that during the baseline period (FDR-adjusted $p$-value $<0.001$ ). 
Table 9. Significant kinesthesia-related spectral perturbation across different type of event suggested by post-hoc comparisons

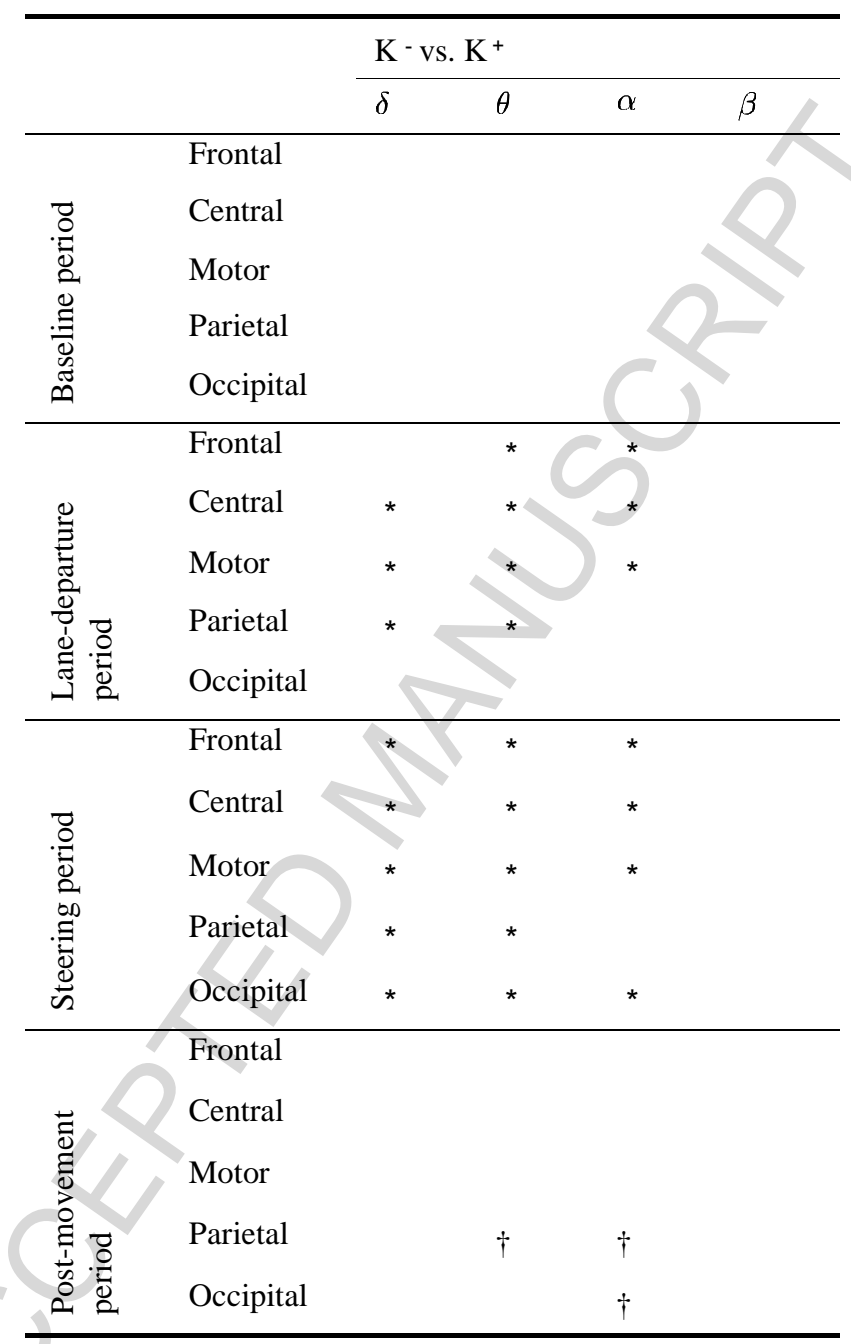

Symbol * indicates that the spectral power of the $\mathrm{K}$ session is significantly larger than that of the $\mathrm{K}^{+}$session. Symbol $\dagger$ indicates that the spectral power of the $\mathrm{K}$ session is significantly less than that of the $\mathrm{K}^{+}$session (FDR-adjusted $p$-value<0.001). 
Table 10. Significant performance-related spectral changes in $\mathrm{K}^{-}$and $\mathrm{K}^{+}$sessions suggested by post-hoc comparisons

\begin{tabular}{|c|c|c|c|c|c|c|c|c|c|c|c|c|c|}
\hline & & \multicolumn{4}{|c|}{ Optimal < suboptimal } & \multicolumn{4}{|c|}{ Suboptimal < poor } & \multicolumn{4}{|c|}{ Optimal < poor } \\
\hline & & $\delta$ & $\theta$ & $\alpha$ & $\beta$ & $\delta$ & $\theta$ & $\alpha$ & $\beta$ & $\delta$ & $\theta$ & $\alpha$ & $\beta$ \\
\hline \multirow[t]{5}{*}{$\mathrm{K}^{-}$} & Frontal & * & * & * & & * & * & * & * & * & * & * & * \\
\hline & Central & & & * & * & * & * & & * & & * & * & * \\
\hline & Motor & & & * & * & * & * & * & & * & * & * & * \\
\hline & Parietal & & & * & * & * & * & & & & * & * & * \\
\hline & Occipital & * & * & * & * & * & * & & * & * & * & * & * \\
\hline \multirow[t]{5}{*}{$\mathrm{K}^{+}$} & Frontal & * & * & * & * & * & & & * & * & * & * & * \\
\hline & Central & * & * & * & * & * & & & * & * & * & * & * \\
\hline & Motor & * & * & & * & * & & & * & * & * & * & * \\
\hline & Parietal & & & * & * & * & & * & * & * & * & * & * \\
\hline & Occipital & * & * & * & * & * & * & * & & * & * & * & * \\
\hline
\end{tabular}

Symbol * refers to a situation in which the difference of power spectra between groups (i.e. optimal<suboptimal, suboptimal<poor, and optimal<poor) is statistically significant (FDR-adjusted $p$ value $<0.001)$. 
Table 11. Significant kinesthesia-related spectral perturbation across different levels of performance suggested by posthoc comparisons

\begin{tabular}{|c|c|c|c|c|c|}
\hline & & \multicolumn{4}{|c|}{$\mathrm{K}^{-}$vs. $\mathrm{K}^{+}$} \\
\hline & & $\delta$ & $\theta$ & $\alpha$ & $\beta$ \\
\hline \multirow[t]{5}{*}{ Optimal } & Frontal & & & & \\
\hline & Central & * & * & A & \\
\hline & Motor & * & * & & \\
\hline & Parietal & & & & \\
\hline & Occipital & & & & \\
\hline \multirow[t]{5}{*}{ Suboptimal } & Frontal & & & & $\dagger$ \\
\hline & Central & & & * & $\dagger$ \\
\hline & Motor & & & * & \\
\hline & Parietal & & & $\dagger$ & $\dagger$ \\
\hline & Occipital & & & & $\dagger$ \\
\hline \multirow[t]{5}{*}{ Poor } & Frontal & * & * & * & * \\
\hline & Central & * & * & * & \\
\hline & Motor & * & & * & * \\
\hline & Parietal & * & * & & \\
\hline & Occipital & * & * & & \\
\hline
\end{tabular}

Symbol * indicates that the power spectra of the $\mathrm{K}$ - session is significantly larger than those of the $\mathrm{K}^{+}$session. Symbol $\dagger$ indicates that the power spectra of the $\mathrm{K}$ - session is significantly less than those of the $\mathrm{K}^{+}$session (FDR-adjusted $p$-value<0.001). 